\title{
Heat equilibrium distribution in a turbulent flow
}

\author{
Z. Haba \\ Institute of Theoretical Physics, University of Wroclaw, \\ 50-204 Wroclaw, Plac Maxa Borna 9,Poland \\ e-mail:zhab@ift.uni.wroc.pl \\ PACS:47.10.A-,44.27.+g,47.27.eb
}

\begin{abstract}
We consider a shear flow of a scale invariant Gaussian random velocity field which does not depend on the coordinates in the direction of the flow. We investigate a heat advection coming from a Gaussian random homogeneous source. We discuss a relaxation at large time of a temperature distribution determined by the forced advection-diffusion equation. We represent the temperature correlation functions by means of the FeynmanKac formula. Jensen inequalities are applied for lower and upper bounds on the correlation functions. We show that at finite time there is no velocity dependence of long range temperature correlations (low momentum asymptotics) in the direction of the flow but the equilibrium heat distribution has large distance correlations (low momentum behavior) with an index depending on the scaling index of the random flow and of the index of the random forcing. If the velocity has correlations growing with the distance ( a turbulent flow) then the large distance correlations depend in a crucial way on the scaling index of the turbulent flow. In such a
\end{abstract}


case the correlations increase in the direction of the flow and decrease in the direction perpendicular to the flow making the stream of heat more coherent.

\section{Introduction}

We investigate a heat advection in a random flow which is supposed to be "turbulent". The turbulence is a complex phenomenon difficult to define and avoiding a description in precise mathematical terms. The complexity of turbulence can be related to its dependence on the length scale relevant for undergoing experiments. In this paper we apply only some aspects of the turbulent flow: randomness of the velocity field, its self-similarity and long range correlations . The appearance of the turbulence should have an impact on transport phenomena described by an advection-diffusion equation of a passive scalar [1]. Such an equation can describe a transport of heat, a mass or some impurities. We are interested in the equilibrium distribution of solutions of the random advectiondiffusion equation. The equilibrium is possible only under an external forcing (a heat source). We are interested in the equilibrium distribution at all scales. Such an equilibrium will depend on the forcing. The universality is possible only in the inertial range [2][3] [4] where the external forcing should not be relevant(see ref.[5] for some recent shear flow experiments). Although the precise equilibrium distribution depends on the form of the forcing the asymptotic behavior of correlation functions depends solely on the asymptotic behavior of the random forcing. We investigate the way the long range correlations of the fluid velocity influence the long range correlations of the temperature.

We assume that there is a distinguished direction of the fluid velocity $\mathbf{V}$. 
We make a decomposition $X=(\mathbf{x}, \mathbf{z}) \in R^{D}$ with $\mathbf{x} \in R^{d}$ and $\mathbf{z} \in R^{D-d}$; $\mathbf{V}(\tau, \mathbf{x})$ depends only on $\mathbf{x} \in R^{d}$ and has the non-vanishing components only in $R^{D-d}$ (in such a case it satisfies automatically $\nabla \mathbf{V}=0$;for physical applications $D=3$ and $d=2$ or $d=1$ ). As a typical example we could consider a fluid flow $V_{z}(x, y)$ in the direction of the $z$-axis which does not depend on $z$. We can impose such an anisotropy of the flow by an external force $\mathbf{R}$ which depends only on $\mathbf{x}$ and has non-zero components solely in the $\mathbf{z}$ direction. So, we consider the Navier-Stokes equation with such a random force $\mathbf{R}$

$$
\partial_{t} \mathbf{V}+\mathbf{V} \nabla \mathbf{V}-\nu \triangle \mathbf{V}=\mathbf{R}
$$

The $(\mathbf{0}, \mathbf{V}(\mathbf{x}))$ solution of the Navier-Stokes equation is the solution of the linear equation ( for the $\mathbf{z}$-component)

$$
\partial_{t} \mathbf{V}-\nu \triangle_{\mathbf{x}} \mathbf{V}=\mathbf{R}
$$

( together with a zero solution for the $\mathbf{x}$-component). By a proper choice of the external force $\mathbf{R}$ we can simulate a large class of $\mathbf{x}$-dependent flows.

In secs.2-3 we discuss the advection-diffusion equation, the random velocity and a random forcing. The advection-diffusion equation can be solved by means of the Feynman-Kac formula. The Feynman-Kac solution has already been discussed by other authors [6]-[7]. These authors have been interested in the asymptotic behavior of the advection-diffusion equation without forcing. Our main interest (secs.4-5) is in the asymptotic behavior for large time and distances of correlation functions of the temperature field resulting from the advection-diffusion equation with forcing describing the heat injection. First, in sec.3 we simulate forcing by a constant gradient term in the temperature. We obtain a simple soluble model of advection illustrating some general features. 
In general, we can obtain some lower and upper bounds on the correlation functions by means of the Jensen inequalities (sec.5). For the sake of simplicity we concentrate on the two-point correlations. In sec.6 we show how our methods can be extended to multi-point correlations. We obtain asymptotic behavior of the Fourier transform of the correlation functions for small and large momenta. We compare our methods and results (in secs.4-6 and in the Appendix B) with an exactly soluble model of Kraichnan [8][4][9] (defined by a velocity field which is a white noise in time). The random advection is closely connected with a diffusion. In fact, under some natural assumptions random advection enforces diffusion [10][11][12] and vice versa the diffusion can be expressed as a white noise advection [13]. However, when we choose no diffusion (zero molecular diffusivity) in the initial equation of advection describing the temperature evolution then we obtain a model of advection (discussed in Appendix A) as a limit of the solution of the random advection-diffusion equation. The limit of zero molecular diffusivity has been discussed earlier in refs.[14][15].

In the text some positive constants arise (denoted usually as $K, c_{1}$, etc.) which are not described at each case and are not related one to another.

\section{The advection-diffusion equation}

We consider the advection in a random velocity field $\mathbf{V}$ ( described in the Introduction) forced by a random source $f$

$$
\partial_{\tau} \theta_{\tau}+\mathbf{V} \nabla \theta_{\tau}-\frac{\mu^{2}}{2} \triangle \theta_{\tau}=f
$$

where $\mu^{2}$ is the molecular diffusivity. If the random velocity $\mathbf{V}$ has correlation functions singular at small time then eq.(1) needs a careful interpretation. If the singularity of the velocity's covariance is of the form $\delta\left(t-t^{\prime}\right) D\left(\mathbf{x}-\mathbf{x}^{\prime}\right)$ then 
there are two standard interpretations either Ito or Stratonovitch [16] [17]. The difference between them in eq.(1) is $\frac{1}{2} D(\mathbf{0}) \nabla_{\mathbf{z}}^{2} \theta$. Hence, choosing one of them will change only the diffusion constant. We choose the Stratonovitch interpretation throughout the paper and also in the Appendix B.

First, let us consider $\mathbf{V}=0$ and $f=0$. Let $N$ be a (deterministic) solution of the heat equation

$$
\partial_{\tau} N_{\tau}-\frac{\mu^{2}}{2} \triangle N_{\tau}=0
$$

We expand $\theta$ around the solution $N$ of the diffusion equation

$$
\theta=T+N
$$

(if the mean value of $\mathbf{V}$ is zero then $T$ describes fluctuations of the temperature). From eq.(1)

$$
\partial_{\tau} T_{\tau}+\mathbf{V} \nabla T_{\tau}-\frac{\mu^{2}}{2} \triangle T_{\tau}=F
$$

where

$$
F=f-\mathbf{V} \nabla N_{\tau}
$$

As the simplest example of a physical relevance we consider the mean gradient $[18][19]$

$$
N=-\mathbf{g X}
$$

where $\mathbf{g}$ is a constant vector. The mean gradient is a stationary solution of the heat equation between two planes kept at fixed temperatures. For such a static solution

$$
F=f+\mathbf{V g}
$$

We can see that even if $f=0$ then $F$ is non-trivial. This is a frequent realization of an advection in experiments [20][21]. In such a case the source $F$ has the same distribution as the velocity. A constant mean gradient is distinguishing 
a direction in space. It breaks the rotational symmetry. As a model we could consider $\mathbf{g}=\left(0,0, g_{z}\right)$ and $\mathbf{V}=\left(0,0, V_{z}\right)$.

We define the spectral measure $\rho$ of the temperature $T$ which is directly measurable in experiments [22]

$$
\begin{aligned}
& \left\langle T_{\tau}(\mathbf{x}, \mathbf{z}) T_{\tau}\left(\mathbf{x}^{\prime}, \mathbf{z}^{\prime}\right)\right\rangle-\left\langle T_{\tau}(\mathbf{x}, \mathbf{z})\right\rangle\left\langle T_{\tau}\left(\mathbf{x}^{\prime}, \mathbf{z}^{\prime}\right)\right\rangle \\
& =\int d \mathbf{k} d \mathbf{p} \exp \left(i \mathbf{k}\left(\mathbf{x}-\mathbf{x}^{\prime}\right)+i \mathbf{p}\left(\mathbf{z}-\mathbf{z}^{\prime}\right)\right) \rho_{\tau}(\mathbf{k}, \mathbf{p})
\end{aligned}
$$

We have

$$
\begin{aligned}
& \int d \mathbf{x}\left(\left\langle\tilde{T}_{\tau}(\mathbf{x}, \mathbf{p}) \tilde{T}_{\tau}\left(\mathbf{x}^{\prime}, \mathbf{p}^{\prime}\right)\right\rangle-\left\langle\tilde{T}_{\tau}(\mathbf{x}, \mathbf{p})\right\rangle\left\langle\tilde{T}_{\tau}\left(\mathbf{x}^{\prime}, \mathbf{p}^{\prime}\right)\right\rangle\right) \\
& =\delta\left(\mathbf{p}+\mathbf{p}^{\prime}\right) \rho_{\tau}(\mathbf{0}, \mathbf{p})
\end{aligned}
$$

and

$$
\begin{gathered}
\left\langle\tilde{T}_{\tau}(\mathbf{x}, \mathbf{p}) \tilde{T}_{\tau}\left(\mathbf{x}, \mathbf{p}^{\prime}\right)\right\rangle-\left\langle\tilde{T}_{\tau}(\mathbf{x}, \mathbf{p})\right\rangle\left\langle\tilde{T}_{\tau}(\mathbf{x}, \mathbf{p})\right\rangle=\delta\left(\mathbf{p}+\mathbf{p}^{\prime}\right) \int d \mathbf{k} \rho_{\tau}(\mathbf{k}, \mathbf{p}) \\
\left\langle T_{\tau}(\mathbf{x}, \mathbf{z}) T_{\tau}(\mathbf{x}, \mathbf{z})\right\rangle-\left\langle T_{\tau}(\mathbf{x}, \mathbf{z})\right\rangle\left\langle T_{\tau}(\mathbf{x}, \mathbf{z})\right\rangle=\int d \mathbf{p} \int d \mathbf{k} \rho_{\tau}(\mathbf{k}, \mathbf{p})
\end{gathered}
$$

When the spectral function has singularities at low momenta then the Fourier transform in eq.(6) may need a careful definition in the sense of generalized functions. Instead of the correlation functions of $T_{\tau}(\mathbf{x}, \mathbf{y})$ we could consider the structure functions

$$
\mathcal{G}_{\tau}^{(2 n)}(\mathbf{x}, \mathbf{z})=\left\langle\left(T_{\tau}(\mathbf{0}, \mathbf{0})-\left\langle T_{\tau}(\mathbf{0}, \mathbf{0})\right\rangle-T_{\tau}(\mathbf{x}, \mathbf{z})+\left\langle T_{\tau}(\mathbf{x}, \mathbf{z})\right\rangle\right)^{2 n}\right\rangle
$$

For $n=1$ we have

$$
\mathcal{G}_{\tau}^{(2)}(\mathbf{x}, \mathbf{z})=2 \int d \mathbf{k} d \mathbf{p} \rho_{\tau}(\mathbf{k}, \mathbf{p})(1-\exp (i \mathbf{k x}+i \mathbf{p z}))
$$

$\mathcal{G}_{\tau}^{(2)}$ scales in the same way as $\langle T T\rangle$ but has better infrared behaviour. The structure functions $\mathcal{G}^{(2 n)}$ are expressed by the correlation functions of the Fourier transforms of $T_{\tau}$. 
It can be seen that the spectral measure $\rho$ of the temperature $T$ depends on the spectral measure of the source $f$ and the scaling properties of the random velocity field.

\section{Gaussian model of the shear flow}

We decompose the fluid velocity

$$
\mathbf{V}=\mathbf{U}+\mathbf{v}
$$

into the mean value $\mathbf{U}$ and random fluctuations $\mathbf{v}$. We assume that the velocity $\mathbf{v}$ is a Gaussian Euclidean $R^{d}$ invariant random field with the mean zero and the covariance

$$
\left\langle v_{j}(s, \mathbf{x}) v_{k}\left(s^{\prime}, \mathbf{x}^{\prime}\right)\right\rangle=G_{j k}\left(s-s^{\prime}, \mathbf{x}, \mathbf{x}^{\prime}\right)
$$

where $j, k=d+1, \ldots, D$. For the sake of simplicity of the arguments we shall sometimes separate the time-dependence choosing $G$ of the product form $\Gamma D$. If $\mathrm{G}$ is a decaying function of the distance $\left|\mathbf{x}-\mathbf{x}^{\prime}\right|$ then a model of the vector field $\mathbf{v}$ can be determined by a translation invariant $\mathrm{G}$, e.g.,

$G_{j k}\left(s-s^{\prime}, \mathbf{x}, \mathbf{x}^{\prime}\right) \equiv \delta_{j k} \Gamma\left(s-s^{\prime}\right) D\left(\mathbf{x}-\mathbf{x}^{\prime}\right)=\delta_{j k} \Gamma\left(s-s^{\prime}\right) \int d \mathbf{p} \exp \left(i \mathbf{p}\left(\mathbf{x}-\mathbf{x}^{\prime}\right)\right) \tilde{D}(\mathbf{p})$

where $\tilde{D}$ is a locally integrable function.

In a description of the turbulence we consider growing long range correlations. In such a case $G$ cannot be translation invariant. We consider a model with Euclidean $R^{d}$ invariant correlation functions of $\mathbf{v}(\mathbf{x})-\mathbf{v}\left(\mathbf{x}^{\prime}\right)$. Then

$$
G_{j k}\left(s-s^{\prime}, \mathbf{x}, \mathbf{x}^{\prime}\right)=\delta_{j k} \Gamma\left(s-s^{\prime}\right)\left(|\mathbf{x}|^{2 \beta}+\left|\mathbf{x}^{\prime}\right|^{2 \beta}-\left|\mathbf{x}-\mathbf{x}^{\prime}\right|^{2 \beta}\right)
$$

This $G$ is positive definite if $\Gamma$ is positive definite and $0<\beta<1$ (the covariance (10) determines Levy's model [23] of the Brownian motion depending 
on $d$-parameters ). When $2 \beta<2$ then the vector field $\mathbf{v}(\mathbf{x})$ does not satisfy the Lipschitz condition. In such a case we could expect difficulties with the uniqueness of the flow and the uniqueness of the solution of eq.(1) at $\mu=0$. Fortunately, a definition of the unique solution of eq.(1) in a weak probabilistic sense is possible [24][25] even without the Lipschitz condition.

The source $f$ is an independent Gaussian field with the covariance

$$
\left\langle f(s, \mathbf{x}, \mathbf{z}) f\left(s^{\prime}, \mathbf{x}^{\prime}, \mathbf{z}^{\prime}\right)\right\rangle=M\left(s-s^{\prime}, \mathbf{x}-\mathbf{x}^{\prime}, \mathbf{z}-\mathbf{z}^{\prime}\right)
$$

We take the Fourier transform of eq.(3) in the $\mathbf{z}$ variable. Then, this equation reads

$$
\partial_{\tau} \tilde{T}_{\tau}(\mathbf{x}, \mathbf{p})+\left(i \mathbf{p} \mathbf{V}(\tau, \mathbf{x})+\frac{\mu^{2} \mathbf{p}^{2}}{2}-\frac{\mu^{2}}{2} \triangle_{\mathbf{x}}\right) \tilde{T}_{\tau}(\mathbf{x}, \mathbf{p})=\tilde{F}(\tau, \mathbf{x}, \mathbf{p})
$$

We apply the Feynman-Kac formula [17] in order to express the solution of eq.(12) with the initial condition $T_{0} \in L^{2}(d X)$ in the form (the uniqueness of the solution is discussed in [24][25])

$$
\begin{aligned}
& \tilde{T}_{\tau}(\mathbf{x}, \mathbf{p})=\exp \left(-\frac{\mu^{2} \mathbf{p}^{2} \tau}{2}\right) E\left[\exp \left(-i \mathbf{p} \int_{0}^{\tau} \mathbf{V}(\tau-s, \mathbf{x}+\mu \mathbf{b}(s)) d s\right) \tilde{T}_{0}(\mathbf{x}+\mu \mathbf{b}(\tau), \mathbf{p})\right]+ \\
& \int_{0}^{\tau} d t \exp \left(-\frac{\mu^{2} \mathbf{p}^{2}(\tau-t)}{2}\right) E\left[\exp \left(-i \mathbf{p} \int_{0}^{\tau-t} \mathbf{V}(\tau-s, \mathbf{x}+\mu \mathbf{b}(s)) d s\right) \tilde{F}(t, \mathbf{x}+\mu \mathbf{b}(\tau-t), \mathbf{p})\right]
\end{aligned}
$$

In eq.(13) $b_{j}(j=1,2, \ldots, d)$ is the Brownian motion defined as the Gaussian process with the covariance [17]

$$
E\left[b_{j}(s) b_{k}(t)\right]=\delta_{j k} \min (s, t)
$$

We are interested in the equilibrium distribution of $T_{\tau}$, i.e., in the limit $\tau \rightarrow \infty$. When $\tau \rightarrow \infty$ and $T_{0} \in L^{2}(d X)$ then the first term in eq.(13) is vanishing. For this reason we may set $T_{0}=0$ from the beginning. The stationary solutions $N$ being harmonic functions are not square integrable in $R^{D}$. Admitting such 
functions as initial conditions we could regain the solution $N$ from eq.(13) (with $F=0$ ). In particular, the mean gradient (4) comes from a generalized function $\tilde{T}_{0}$ with its support concentrated at $\mathbf{p}=0$.

Before discussing more general correlations let us consider the constant mean gradient (eqs.(4)-(5)) with $f=0$ and $F=\mathbf{g V}$. Then, from eq.(13) (with $T_{0}=0$ )

$$
\tilde{T}_{\tau}(\mathbf{x}, \mathbf{p})=\delta(\mathbf{p}) E\left[\int_{0}^{\tau} d t \mathbf{g} \mathbf{V}(t, \mathbf{x}+\mu \mathbf{b}(\tau-t))\right]
$$

We shall see that some properties of the general advection (3) appear already at the level of the simple model (14). It follows from eq.(14) that

$$
\left\langle T_{\tau}(\mathbf{X})\right\rangle=\delta(\mathbf{p}) E\left[\int_{0}^{\tau} d t \mathbf{g} \mathbf{U}(t, \mathbf{x}+\mu \mathbf{b}(\tau-t))\right]
$$

and

$$
\begin{aligned}
& \left\langle\tilde{T}_{\tau}(\mathbf{x}, \mathbf{p}) \tilde{T}_{\tau}\left(\mathbf{x}^{\prime}, \mathbf{p}^{\prime}\right)\right\rangle-\left\langle\tilde{T}_{\tau}(\mathbf{x}, \mathbf{p})\right\rangle\left\langle\tilde{T}_{\tau}\left(\mathbf{x}^{\prime}, \mathbf{p}^{\prime}\right)\right\rangle=\delta(\mathbf{p}) \delta\left(\mathbf{p}^{\prime}\right) \\
& \int_{0}^{\tau} d t \int_{0}^{\tau} d t^{\prime} E\left[\mathbf{g} G\left(t-t^{\prime}, \mathbf{x}-\mathbf{x}^{\prime}+\mu \mathbf{b}(\tau-t)-\mu \mathbf{b}^{\prime}\left(\tau-t^{\prime}\right)\right) \mathbf{g}\right]
\end{aligned}
$$

We calculate the integral over time. First, if the covariance $G$ is time-independent (a steady flow) then

$$
\begin{aligned}
& \left\langle T_{\tau}(\mathbf{x}, \mathbf{z}) T_{\tau}\left(\mathbf{x}^{\prime}, \mathbf{z}^{\prime}\right)\right\rangle-\left\langle T_{\tau}(\mathbf{X})\right\rangle\left\langle T_{\tau}\left(\mathbf{X}^{\prime}\right)\right\rangle \\
& =4 \mu^{-4} \int d \mathbf{k} \exp \left(i \mathbf{k}\left(\mathbf{x}-\mathbf{x}^{\prime}\right)\right) \mathbf{g} \tilde{G}(\mathbf{k}) \mathbf{g}|\mathbf{k}|^{-4}\left(1-\exp \left(-\frac{\mu^{2}}{2} \mathbf{k}^{2} \tau\right)\right)^{2}
\end{aligned}
$$

Next, let us consider

$$
G\left(t-t^{\prime}, \mathbf{x}-\mathbf{x}^{\prime}\right)=\delta\left(t-t^{\prime}\right) D\left(\mathbf{x}-\mathbf{x}^{\prime}\right)
$$

The covariance (16) does not have any physical foundations but the virtue of the assumption (16) is the solubility of the model (3) [8](the Kraichnan model) in the sense that one can obtain a closed set of partial differential equations for the correlation functions (see the Appendix B). In our simplified version (14)

$$
\begin{aligned}
& \left\langle T_{\tau}(\mathbf{x}, \mathbf{z}) T_{\tau}\left(\mathbf{x}^{\prime}, \mathbf{z}^{\prime}\right)\right\rangle-\left\langle T_{\tau}(\mathbf{X})\right\rangle\left\langle T_{\tau}\left(\mathbf{X}^{\prime}\right)\right\rangle \\
& =\mu^{-2} \int d \mathbf{k} \exp \left(i \mathbf{k}\left(\mathbf{x}-\mathbf{x}^{\prime}\right)\right) \mathbf{g} \tilde{D}(\mathbf{k}) \mathbf{g}|\mathbf{k}|^{-2}\left(1-\exp \left(-\mu^{2} \mathbf{k}^{2} \tau\right)\right)
\end{aligned}
$$


If the $\mathbf{v}$ correlations are growing as in eq.(10) then the expression (17) can be infrared divergent (especially at $\tau=\infty$ ). In such a case we should rather consider

$$
\begin{aligned}
& \left\langle\left(T_{\tau}(\mathbf{0}, \mathbf{0})-\left\langle T_{\tau}(\mathbf{0}, \mathbf{0})\right\rangle-T_{\tau}(\mathbf{x}, \mathbf{z})+\left\langle T_{\tau}(\mathbf{x}, \mathbf{z})\right\rangle\right)^{2}\right\rangle \\
& =8 \mu^{-4} \int d \mathbf{k}(1-\exp (i \mathbf{k x})) \tilde{G}(\mathbf{k})|\mathbf{k}|^{-4}\left(1-\exp \left(-\frac{\mu^{2}}{2} \mathbf{k}^{2} \tau\right)\right)^{2}
\end{aligned}
$$

In general, let

$$
G\left(t-t^{\prime}, \mathbf{x}-\mathbf{x}\right)=\int d \omega d \mathbf{k} \tilde{G}(\omega, \mathbf{k}) \exp \left(i \omega\left(t-t^{\prime}\right)+i \mathbf{k}\left(\mathbf{x}-\mathbf{x}^{\prime}\right)\right)
$$

then

$$
\begin{aligned}
& \left\langle\tilde{T}_{\tau}(\mathbf{x}, \mathbf{p}) \tilde{T}_{\tau}\left(\mathbf{x}^{\prime}, \mathbf{p}^{\prime}\right)\right\rangle-\left\langle\tilde{T}_{\tau}(\mathbf{x}, \mathbf{p})\right\rangle\left\langle T_{\tau}\left(\mathbf{x}^{\prime}, \mathbf{p}^{\prime}\right)\right\rangle=\delta(\mathbf{p}) \delta\left(\mathbf{p}^{\prime}\right) \\
& \int_{0}^{\tau} d t \int_{0}^{\tau} d t^{\prime} \int d \mathbf{k g} \tilde{G}\left(t-t^{\prime}, \mathbf{k}\right) \mathbf{g} \exp \left(i \mathbf{k}\left(\mathbf{x}-\mathbf{x}^{\prime}\right)-\frac{1}{2} \mu^{2} \mathbf{k}^{2}\left(2 \tau-t-t^{\prime}\right)\right)
\end{aligned}
$$

After the time integration

$$
\begin{aligned}
& \left\langle T_{\tau}(\mathbf{x}, \mathbf{z}) T_{\tau}\left(\mathbf{x}^{\prime}, \mathbf{z}^{\prime}\right)\right\rangle-\left\langle T_{\tau}(X)\right\rangle\left\langle T_{\tau}\left(X^{\prime}\right)\right\rangle=\int d \mathbf{k} d \omega \exp \left(i \mathbf{k}\left(\mathbf{x}-\mathbf{x}^{\prime}\right)\right) \mathbf{g} \tilde{G}(\omega, \mathbf{k}) \mathbf{g} \\
& \left(\frac{1}{4} \mu^{4}|\mathbf{k}|^{4}+\omega^{2}\right)^{-1}\left|1-\exp \left(-\frac{1}{2} \mu^{2} \mathbf{k}^{2} \tau-i \omega \tau\right)\right|^{2}
\end{aligned}
$$

We assume that $G$ is scale invariant

$$
G(c t, \lambda \mathbf{x})=c^{-\alpha} \lambda^{-2 \gamma} G(t, \mathbf{x})
$$

( $\alpha+\gamma<1$ if the time integral in eq.(14) is to be finite). This assumption has simple consequences for the heat transport. It may be not exact in mathematical models. As an example, for the shear flow solution of the Navier-Stokes equation discussed in the Introduction if $C_{j l}(\omega, \mathbf{k})$ is the spectral function of the force distribution $\mathbf{R}$ then the spectral function of the stationary velocity distribution (obtained as a solution of the Navier-Stokes equation with the initial condition at $t_{0}$ and then letting $\left.t_{0} \rightarrow-\infty\right)$ is

$$
\tilde{G}_{j l}(\omega, \mathbf{k})=C_{j l}(\omega, \mathbf{k})\left(\left(\frac{\nu}{2} \mathbf{k}^{2}\right)^{2}+\omega^{2}\right)^{-1}
$$


We must choose a specific $C$ in order to obtain a scale invariant $\tilde{G}$.

We can see from eqs.(15)-(20) that at finite $\tau$ the large distance behavior of the temperature correlations is the same as that of the velocity correlations because the behavior of $\rho_{\tau}$ for small momenta does not change. However,if $\langle\mathbf{v}(\mathbf{x}) \mathbf{v}(\mathbf{0})\rangle \simeq|\mathbf{x}|^{2 \beta}$ then at $\tau=\infty$ for a steady flow we obtain in eq.(18)

$$
\left\langle\left(T_{\infty}(\mathbf{x}, \mathbf{z})-\left\langle T_{\infty}(\mathbf{x}, \mathbf{z})\right\rangle-T_{\infty}(\mathbf{0}, \mathbf{0})+\left\langle T_{\infty}(\mathbf{0}, \mathbf{0})\right\rangle\right)^{2}\right\rangle \simeq|\mathbf{x}|^{2 \beta+4}
$$

and for the Kraichnan model [8]

$$
\left\langle\left(T_{\infty}(\mathbf{x}, \mathbf{z})-\left\langle T_{\infty}(\mathbf{x}, \mathbf{z})\right\rangle-T_{\infty}(\mathbf{0}, \mathbf{0})+\left\langle T_{\infty}(\mathbf{0}, \mathbf{0})\right\rangle\right)^{2}\right\rangle \simeq|\mathbf{x}|^{2 \beta+2}
$$

in eq.(17) . For a general time dependent $G(t, \mathbf{x})$ of the form (19) we shall have the $|\mathbf{x}|^{2 \beta-2 \alpha+4}$ behavior of the structure functions $S_{\infty}^{(2)}$ in eq.(20) if $G$ scales as in eq.(21) $(\gamma=-\beta)$. We can establish the behavior for large $\mathbf{x}-\mathbf{x}^{\prime}$ by means of a change of variables in the integrals (15)-(20) $\mathbf{k}=\tilde{\mathbf{k}}\left|\mathbf{x}-\mathbf{x}^{\prime}\right|^{-1}$ and $\omega=\tilde{\omega}\left|\mathbf{x}-\mathbf{x}^{\prime}\right|^{-2}$ and an estimate of the remainder. Note that the long range correlations of the velocity field $(\gamma<0)$ lead to an increase of the temperature correlations.

\section{Gaussian white noise source}

In this section we consider $F=f$ as a Gaussian random field independent of v. Estimates on the equilibrium distribution are simplified if the sources at different times are independent

$$
M\left(t-t^{\prime}, \mathbf{x}-\mathbf{x}^{\prime}, \mathbf{z}-\mathbf{z}^{\prime}\right)=\delta\left(t-t^{\prime}\right) m\left(\mathbf{x}-\mathbf{x}^{\prime}, \mathbf{z}-\mathbf{z}^{\prime}\right)
$$

We assume the form (23) of $M$ as a technical simplification. This is a mathematical idealization still justified by an application of physical sources of heat (as heat injections independent at each time). 
For a lower bound we need an assumption that the dependence on $\mathbf{x}-\mathbf{x}^{\prime}$ is of the form of the Laplace transform (such an assumption includes the scale invariant distributions $m$ which do not increase at large distances) either in the form

$$
\begin{aligned}
& m\left(\mathbf{x}-\mathbf{x}^{\prime}, \mathbf{z}-\mathbf{z}^{\prime}\right) \equiv m_{1}\left(\mathbf{x}-\mathbf{x}^{\prime}\right) m_{0}\left(\mathbf{z}-\mathbf{z}^{\prime}\right) \\
& =\int d \mathbf{k} d \mathbf{p} \exp \left(i \mathbf{k}\left(\mathbf{x}-\mathbf{x}^{\prime}\right)+i \mathbf{p}\left(\mathbf{z}-\mathbf{z}^{\prime}\right)\right) \tilde{m}_{1}(\mathbf{k}) \tilde{m}_{0}(\mathbf{p}) \\
& =\int_{0}^{\infty} d a \nu_{1}(a) \exp \left(-a\left|\mathbf{x}-\mathbf{x}^{\prime}\right|^{2}\right) m_{0}\left(\mathbf{z}-\mathbf{z}^{\prime}\right)
\end{aligned}
$$

or in the Euclidean invariant way

$$
\begin{aligned}
& m\left(\mathbf{x}-\mathbf{x}^{\prime}, \mathbf{z}-\mathbf{z}^{\prime}\right)=\int_{0}^{\infty} d a \nu(a) \exp \left(-a\left(\left|\mathbf{x}-\mathbf{x}^{\prime}\right|^{2}+\left|\mathbf{z}-\mathbf{z}^{\prime}\right|^{2}\right)\right) \\
& \equiv \int_{0}^{\infty} d a \int d \mathbf{p} \exp \left(i \mathbf{p}\left(\mathbf{z}-\mathbf{z}^{\prime}\right)\right) \exp \left(-a\left|\mathbf{x}-\mathbf{x}^{\prime}\right|^{2}\right) \nu(a, \mathbf{p})
\end{aligned}
$$

In eqs.(24)-(25) $\nu_{1}$ and $\nu$ are non-negative functions.

$\mathbf{v}$ in eq.(13) enters $T_{\tau}$ in the form

$$
\exp (i \mathbf{v}(\mathbf{J}))
$$

where

$$
\mathbf{v}(\mathbf{J})=\int d \mathbf{u} \int_{0}^{\tau} d s \mathbf{v}(s, \mathbf{u}) \mathbf{J}(s, \mathbf{u})
$$

with

$$
\mathbf{J}(s, \mathbf{u})=-\theta(s) \mathbf{p} \delta(\mathbf{u}-\mathbf{x}-\mu \mathbf{b}(\tau-s))
$$

It follows that the expectation values of $n$ products of $T_{\tau}$ are expressed by

$$
\left\langle\exp \left(i \mathbf{v}\left(\mathbf{J}_{n}\right)\right)\right\rangle=S\left(\mathbf{J}_{n}\right)
$$

where $S(\mathbf{J})$ is the characteristic function of the random field $\mathbf{v}$. For a Gaussian random field

$$
S(\mathbf{J})=\exp \left(-\frac{1}{2} \mathbf{J} G \mathbf{J}\right)
$$


Let us note that because of the translation invariance in the $\mathbf{z}$ variable of the source $f$ we have a conservation of momenta

$$
\left\langle\tilde{T}_{\tau}\left(\mathbf{x}_{1}, \mathbf{p}_{1}\right) \ldots . \tilde{T}_{\tau}\left(\mathbf{x}_{n}, \mathbf{p}_{n}\right)\right\rangle=\delta\left(\mathbf{p}_{1}+\ldots+\mathbf{p}_{n}\right) \mathcal{H}
$$

The correlation functions (27) are expressed by the characteristic function (26) with $\mathbf{J}_{n}$ satisfying the condition (for $n>1$ )

$$
\int \mathbf{J}_{n}(s, \mathbf{u}) d \mathbf{u}=0
$$

It follows that in the Gaussian case with the covariance (10) the part of $G$ which is not translation invariant does not contribute to the correlation functions.

We calculate the equal time expectation values of $T_{\tau}$ ( eq.(13)with the zero initial condition) under the assumption that the random fields $f$ and $\mathbf{v}$ are independent

$$
\begin{aligned}
& \left\langle\tilde{T}_{\tau}(\mathbf{x}, \mathbf{p}) \tilde{T}_{\tau}\left(\mathbf{x}^{\prime}, \mathbf{p}^{\prime}\right)\right\rangle=\delta\left(\mathbf{p}+\mathbf{p}^{\prime}\right) \int_{0}^{\tau} d t \exp \left(-\mu^{2} \mathbf{p}^{2}(\tau-t)\right) \\
& E\left[\exp \left(-i \mathbf{p} \int_{0}^{\tau-t} \mathbf{U}(\tau-s, \mathbf{x}+\mu \mathbf{b}(s)) d s\right)\right. \\
& \left.\tilde{m}\left(\mathbf{x}-\mathbf{x}^{\prime}+\mu \mathbf{b}(\tau-t)-\mu \mathbf{b}^{\prime}(\tau-t), \mathbf{p}\right) S\left(\mathbf{J}_{2}\right)\right]
\end{aligned}
$$

where

$$
\mathbf{J}_{2}(\mathbf{u})=\mathbf{p} \theta(s) \delta(\mathbf{u}-\mathbf{x}-\mathbf{b}(\tau-s))-\mathbf{p} \theta(s) \delta\left(\mathbf{u}-\mathbf{x}^{\prime}-\mathbf{b}^{\prime}(\tau-s)\right)
$$

For the Gaussian field (26)

$$
\begin{aligned}
& S\left(\mathbf{J}_{2}\right)=\exp \left(-\frac{1}{2} \int_{0}^{\tau-t} \int_{0}^{\tau-t} d s d s^{\prime} \mathbf{p} G_{0}\left(s-s^{\prime}, \mu \mathbf{b}(s)-\mu \mathbf{b}\left(s^{\prime}\right)\right) \mathbf{p}\right. \\
& -\frac{1}{2} \int_{0}^{\tau-t} \int_{0}^{\tau-t} d s d s^{\prime} \mathbf{p} G_{0}\left(s-s^{\prime}, \mu \mathbf{b}^{\prime}(s)-\mu \mathbf{b}^{\prime}\left(s^{\prime}\right)\right) \mathbf{p} \\
& \left.+\int_{0}^{\tau-t} \int_{0}^{\tau-t} d s d s^{\prime} \mathbf{p} G_{0}\left(s-s^{\prime}, \mathbf{x}-\mathbf{x}^{\prime}+\mu \mathbf{b}(s)-\mu \mathbf{b}^{\prime}\left(s^{\prime}\right)\right) \mathbf{p}\right)
\end{aligned}
$$

where $G_{0}$ is the translation invariant part of $G$. 
If

$$
|\tilde{m}(\mathbf{x}, \mathbf{p})| \leq K\left|\tilde{m}_{0}\right|(\mathbf{p})
$$

then from $|S(\mathbf{J})| \leq 1$ there follows the bound

$$
\left|\left\langle\tilde{T}_{\tau}(\mathbf{x}, \mathbf{p}) \tilde{T}_{\tau}\left(\mathbf{x}^{\prime}, \mathbf{p}^{\prime}\right)\right\rangle\right| \leq K \delta\left(\mathbf{p}+\mathbf{p}^{\prime}\right)\left|\tilde{m}_{0}\right|(\mathbf{p}) \mu^{-2} \mathbf{p}^{-2}\left(1-\exp \left(-\mu^{2} \mathbf{p}^{2} \tau\right)\right)
$$

For a small $\mathbf{p}$ and a finite $\tau$ the correlations (32) are bounded by $\tau\left|\tilde{m}_{0}\right|(\mathbf{p})$ whereas at $\tau=\infty$ by $\left|\tilde{m}_{0}\right|(\mathbf{p}) \mathbf{p}^{-2}$.

Next, we apply the scale invariance of the Brownian motion

$$
\mathbf{b}(a t)=\sqrt{a} \mathbf{b}(t)
$$

in eq.(29).We write $s=(\tau-t) \sigma$. Then, using the scaling properties (21) and (33) and denoting by $G_{0}$ the translation invariant part of $G$ we can rewrite eqs.(29)-(30) in the form

$$
\begin{aligned}
& \left\langle\tilde{T}_{\tau}(\mathbf{x}, \mathbf{p}) \tilde{T}_{\tau}\left(\mathbf{x}^{\prime}, \mathbf{p}^{\prime}\right)\right\rangle=\delta\left(\mathbf{p}+\mathbf{p}^{\prime}\right) \int_{0}^{\tau} d t \exp \left(-\mu^{2} \mathbf{p}^{2}(\tau-t)\right) \\
& E\left[\exp \left(-i \mathbf{p} \int_{0}^{\tau-t} \mathbf{U}(\tau-s, \mathbf{x}+\mu \mathbf{b}(s)) d s\right) \tilde{m}\left(\mathbf{x}-\mathbf{x}^{\prime}+\mu \sqrt{\tau-t} \mathbf{b}(1)-\mu \sqrt{\tau-t} \mathbf{b}^{\prime}(1), \mathbf{p}\right)\right. \\
& \exp \left(-\frac{1}{2}(\tau-t)^{2-\alpha-\gamma} \int_{0}^{1} \int_{0}^{1} d \sigma d \sigma^{\prime} \mathbf{p} G_{0}\left(\sigma-\sigma^{\prime}, \mu \mathbf{b}(\sigma)-\mu \mathbf{b}\left(\sigma^{\prime}\right)\right) \mathbf{p}\right. \\
& -\frac{1}{2}(\tau-t)^{2-\alpha-\gamma} \int_{0}^{1} \int_{0}^{1} d \sigma d \sigma^{\prime} \mathbf{p} G_{0}\left(\sigma-\sigma^{\prime}, \mu \mathbf{b}^{\prime}(\sigma)-\mu \mathbf{b}^{\prime}\left(\sigma^{\prime}\right)\right) \mathbf{p} \\
& \left.\left.+(\tau-t)^{2-\alpha-\gamma} \int_{0}^{1} \int_{0}^{1} d \sigma d \sigma^{\prime} \mathbf{p} G_{0}\left(\sigma-\sigma^{\prime},(\tau-t)^{-\frac{1}{2}}\left(\mathbf{x}-\mathbf{x}^{\prime}\right)+\mu \mathbf{b}(\sigma)-\mu \mathbf{b}^{\prime}\left(\sigma^{\prime}\right)\right) \mathbf{p}\right)\right]
\end{aligned}
$$

For the Kraichnan model [8] $\Gamma\left(s-s^{\prime}\right)=\delta\left(s-s^{\prime}\right)$ in eqs.(9)-(10), then $\alpha=1$ in eq.(22) and the formula (34) reads

$$
\begin{aligned}
& \left\langle\tilde{T}_{\tau}(\mathbf{x}, \mathbf{p}) \tilde{T}_{\tau}\left(\mathbf{x}^{\prime}, \mathbf{p}^{\prime}\right)\right\rangle=\delta\left(\mathbf{p}+\mathbf{p}^{\prime}\right) \int_{0}^{\tau} d t \exp \left(-\mu^{2} \mathbf{p}^{2}(\tau-t)\right) \\
& E\left[\exp \left(-i \mathbf{p} \int_{0}^{\tau-t} \mathbf{U}(\tau-s, \mathbf{x}+\mu \mathbf{b}(s)) d s\right) \tilde{m}\left(\mathbf{x}-\mathbf{x}^{\prime}+\mu \sqrt{\tau-t} \mathbf{b}(1)-\mu \sqrt{\tau-t} \mathbf{b}^{\prime}(1), \mathbf{p}\right)\right. \\
& \left.\exp \left(-(\tau-t)^{1-\gamma} \mathbf{p} D(\mathbf{0}) \mathbf{p}+(\tau-t)^{1-\gamma} \int_{0}^{1} d \sigma \mathbf{p} D\left((\tau-t)^{-\frac{1}{2}}\left(\mathbf{x}-\mathbf{x}^{\prime}\right)+\mu \mathbf{b}(\sigma)-\mu \mathbf{b}^{\prime}(\sigma)\right) \mathbf{p}\right)\right]
\end{aligned}
$$




\section{Jensen inequalities for the temperature corre- lations}

We are going to estimate the spectral measure (6)-(7) by an application of the Jensen inequality. We can obtain an upper bound on the correlation functions applying the Jensen inequality $\left(\int d \nu \exp f \geq \exp \left(\int d \nu f\right)\right.$ if $\left.\int d \nu=1\right)[26]$ to the time integral in eqs.(29)-(30)

$$
\begin{aligned}
& \left|\left\langle\tilde{T}_{\tau}(\mathbf{x}, \mathbf{p}) \tilde{T}_{\tau}\left(\mathbf{x}^{\prime}, \mathbf{p}^{\prime}\right)\right\rangle\right| \leq \\
& 2 \delta\left(\mathbf{p}+\mathbf{p}^{\prime}\right) \int_{0}^{\tau} d r \int_{0}^{1} d \sigma \int_{0}^{\sigma} d \sigma^{\prime} \exp \left(-\mu^{2} \mathbf{p}^{2} r\right) \\
& E\left[\left|\tilde{m}\left(\mathbf{x}-\mathbf{x}^{\prime}+\mu \sqrt{r} \mathbf{b}(1)-\mu \sqrt{r} \mathbf{b}^{\prime}(1), \mathbf{p}\right)\right|\right. \\
& \exp \left(-\frac{1}{2} r^{2-\alpha-\gamma} \mathbf{p} G_{0}\left(\sigma-\sigma^{\prime}, \mu \mathbf{b}(\sigma)-\mu \mathbf{b}\left(\sigma^{\prime}\right)\right) \mathbf{p}\right. \\
& -\frac{1}{2} r^{2-\alpha-\gamma} \mathbf{p} G_{0}\left(\sigma-\sigma^{\prime}, \mu \mathbf{b}^{\prime}(\sigma)-\mu \mathbf{b}^{\prime}\left(\sigma^{\prime}\right)\right) \mathbf{p} \\
& \left.\left.+r^{2-\alpha-\gamma} \mathbf{p} G_{0}\left(\sigma-\sigma^{\prime}, r^{-\frac{1}{2}}\left(\mathbf{x}-\mathbf{x}^{\prime}\right)+\mu \mathbf{b}(\sigma)-\mu \mathbf{b}^{\prime}\left(\sigma^{\prime}\right)\right) \mathbf{p}\right)\right]
\end{aligned}
$$

Let $p(s, \mathbf{u} ; t, \mathbf{w})$ be the transition function for the Brownian motion to pass from $\mathbf{u}$ at time $s$ to $\mathbf{w}$ at time $t$. Then, the expectation value (36) reads

$$
\begin{aligned}
& \left|\left\langle\tilde{T}_{\tau}(\mathbf{x}, \mathbf{p}) \tilde{T}_{\tau}\left(\mathbf{x}^{\prime}, \mathbf{p}^{\prime}\right)\right\rangle\right| \leq \\
& 2 \delta\left(\mathbf{p}+\mathbf{p}^{\prime}\right) \int d \mathbf{u} d \mathbf{u}^{\prime} d \mathbf{w} d \mathbf{w}^{\prime} \int_{0}^{\tau} d r \int_{0}^{1} d \sigma \int_{0}^{\sigma} d \sigma^{\prime} \exp \left(-\mu^{2} \mathbf{p}^{2} r\right) \\
& p\left(0, \mathbf{0} ; \sigma^{\prime}, \mathbf{u}\right) p\left(\sigma^{\prime}, \mathbf{u} ; \sigma, \mathbf{w}\right) p(\sigma, \mathbf{w} ; 1, \mathbf{z}) p\left(0, \mathbf{0} ; \sigma^{\prime}, \mathbf{u}^{\prime}\right) p\left(\sigma^{\prime}, \mathbf{u}^{\prime} ; \sigma, \mathbf{w}^{\prime}\right) p\left(\sigma, \mathbf{w}^{\prime} ; 1, \mathbf{z}^{\prime}\right) \\
& \left|\tilde{m}\left(\mathbf{x}-\mathbf{x}^{\prime}+\mu \sqrt{r} \mathbf{z}-\mu \sqrt{r} \mathbf{z}^{\prime}, \mathbf{p}\right)\right| \\
& \exp \left(-\frac{1}{2} r^{2-\alpha-\gamma} \mathbf{p} G_{0}\left(\sigma-\sigma^{\prime}, \mu \mathbf{w}-\mu \mathbf{u}\right) \mathbf{p}-\frac{1}{2} r^{2-\alpha-\gamma} \mathbf{p} G_{0}\left(\sigma-\sigma^{\prime}, \mu \mathbf{w}^{\prime}-\mu \mathbf{u}^{\prime}\right) \mathbf{p}\right. \\
& +\frac{1}{2} r^{2-\alpha-\gamma} \mathbf{p} G_{0}\left(\sigma-\sigma^{\prime}, r^{-\frac{1}{2}}\left(\mathbf{x}-\mathbf{x}^{\prime}\right)+\mu \mathbf{w}-\mu \mathbf{u}^{\prime}\right) \mathbf{p} \\
& \left.+\frac{1}{2} r^{2-\alpha-\gamma} \mathbf{p} G_{0}\left(\sigma-\sigma^{\prime}, r^{-\frac{1}{2}}\left(\mathbf{x}-\mathbf{x}^{\prime}\right)+\mu \mathbf{w}^{\prime}-\mu \mathbf{u}\right) \mathbf{p}\right)
\end{aligned}
$$


Till now we have kept the mean velocity $\mathbf{U}$ as an arbitrary non zero function.We can obtain a lower bound only if

$$
\mathbf{U}=0
$$

As claimed by some authors (see ,e.g.,the standard text-book [27]) the mean velocity does not play any essential role in turbulence. So, setting it equal to zero we do not lose much. Moreover, for the lower bound we must assume $m$ of the form $(24)$ (or $(25)$ ) with $\tilde{m}_{0}(\mathbf{p}) \geq 0$. Then, we can apply the Jensen inequality to the expectation value over the Brownian motion

$$
\begin{aligned}
& \left\langle\tilde{T}_{\tau}(\mathbf{x}, \mathbf{p}) \tilde{T}_{\tau}\left(\mathbf{x}^{\prime}, \mathbf{p}^{\prime}\right)\right\rangle \geq \\
& \delta\left(\mathbf{p}+\mathbf{p}^{\prime}\right) \int_{0}^{\tau} d r \exp \left(-\mu^{2} \mathbf{p}^{2} r\right) \int_{0}^{\infty} d a \nu_{1}(a) \\
& \tilde{m}_{0}(\mathbf{p}) \exp E\left[-\frac{1}{2} r^{2-\alpha-\gamma} \int_{0}^{1} \int_{0}^{1} d \sigma d \sigma^{\prime} \mathbf{p} G_{0}\left(\sigma-\sigma^{\prime}, \mu \mathbf{b}(\sigma)-\mu \mathbf{b}\left(\sigma^{\prime}\right)\right) \mathbf{p}\right. \\
& -\frac{1}{2} r^{2-\alpha-\gamma} \int_{0}^{1} \int_{0}^{1} d \sigma d \sigma^{\prime} \mathbf{p} G_{0}\left(\sigma-\sigma^{\prime}, \mu \mathbf{b}^{\prime}(\sigma)-\mu \mathbf{b}^{\prime}\left(\sigma^{\prime}\right)\right) \mathbf{p} \\
& +r^{2-\alpha-\gamma} \int_{0}^{1} \int_{0}^{1} d \sigma d \sigma^{\prime} \mathbf{p} G_{0}\left(\sigma-\sigma^{\prime}, r^{-\frac{1}{2}}\left(\mathbf{x}-\mathbf{x}^{\prime}\right)+\mu \mathbf{b}(\sigma)-\mu \mathbf{b}^{\prime}\left(\sigma^{\prime}\right)\right) \mathbf{p} \\
& \left.-a\left|\mathbf{x}-\mathbf{x}^{\prime}+\mu \sqrt{r} \mathbf{b}(1)-\mu \sqrt{r} \mathbf{b}^{\prime}(1)\right|^{2}\right]
\end{aligned}
$$

For $m$ of the form (25) the inequality (38) reads

$$
\begin{aligned}
& \left\langle\tilde{T}_{\tau}(\mathbf{x}, \mathbf{p}) \tilde{T}_{\tau}\left(\mathbf{x}^{\prime}, \mathbf{p}^{\prime}\right)\right\rangle \geq \\
& \delta\left(\mathbf{p}+\mathbf{p}^{\prime}\right) \int_{0}^{\tau} d r \exp \left(-\mu^{2} \mathbf{p}^{2} r\right) \int_{0}^{\infty} d a \nu(a, \mathbf{p}) \\
& \exp E\left[-\frac{1}{2} r^{2-\alpha-\gamma} \int_{0}^{1} \int_{0}^{1} d \sigma d \sigma^{\prime} \mathbf{p} G_{0}\left(\sigma-\sigma^{\prime}, \mu \mathbf{b}(\sigma)-\mu \mathbf{b}\left(\sigma^{\prime}\right)\right) \mathbf{p}\right. \\
& -\frac{1}{2} r^{2-\alpha-\gamma} \int_{0}^{1} \int_{0}^{1} d \sigma d \sigma^{\prime} \mathbf{p} G_{0}\left(\sigma-\sigma^{\prime}, \mu \mathbf{b}^{\prime}(\sigma)-\mu \mathbf{b}^{\prime}\left(\sigma^{\prime}\right)\right) \mathbf{p} \\
& +r^{2-\alpha-\gamma} \int_{0}^{1} \int_{0}^{1} d \sigma d \sigma^{\prime} \mathbf{p} G_{0}\left(\sigma-\sigma^{\prime}, r^{-\frac{1}{2}}\left(\mathbf{x}-\mathbf{x}^{\prime}\right)+\mu \mathbf{b}(\sigma)-\mu \mathbf{b}^{\prime}\left(\sigma^{\prime}\right)\right) \mathbf{p} \\
& \left.-a\left|\mathbf{x}-\mathbf{x}^{\prime}+\mu \sqrt{r} \mathbf{b}(1)-\mu \sqrt{r} \mathbf{b}^{\prime}(1)\right|^{2}\right]
\end{aligned}
$$

The correlation functions (36)-(39) in general will essentially depend on the source distribution $m$. We consider $m$ such that:i) $m_{1}$ is bounded from above 
by a constant (eq.(31)) and in addition ii) $m_{1}(\mathbf{x})$ is decreasing like a power $2 \Omega$ of $|\mathbf{x}|$. From eq.(32) it follows that under the assumption (31) the limit $\tau \rightarrow \infty$ exists. We wish to estimate the correlation functions at $\tau=\infty$ under various conditions on $m_{1}(\mathbf{x})$. Using the inequality (for $A \geq 0$ )

$2 \exp \left(-\mu^{2} \mathbf{p}^{2} r-A\left(\mathbf{x}-\mathbf{x}^{\prime}, \mathbf{b}\right) r^{2-\alpha-\gamma} \mathbf{p}^{2}\right) \leq \exp \left(-\mu^{2} \mathbf{p}^{2} r\right)+\exp \left(-A\left(\mathbf{x}-\mathbf{x}^{\prime}, \mathbf{b}\right) r^{2-\alpha-\gamma} \mathbf{p}^{2}\right)$

and a change of variables in the $r$-integral in eqs.(36)-(37) $r=t|\mathbf{p}|^{-\frac{2}{2-\alpha-\gamma}}$ we obtain ( when $m_{1}$ is a bounded function (31))

$$
\begin{aligned}
& \left\langle\tilde{T}_{\infty}(\mathbf{x}, \mathbf{p}) \tilde{T}_{\infty}\left(\mathbf{x}, \mathbf{p}^{\prime}\right)\right\rangle \\
& \leq \delta\left(\mathbf{p}+\mathbf{p}^{\prime}\right)\left|\tilde{m}_{0}\right|(\mathbf{p})\left(c_{1} \theta\left(|\mathbf{p}|-\frac{1}{\mu}\right)|\mathbf{p}|^{-2}+c_{2} \theta\left(\frac{1}{\mu}-|\mathbf{p}|\right)|\mathbf{p}|^{-\frac{2}{2-\alpha-\gamma}}\right)
\end{aligned}
$$

where on the rhs of eq.(36) after an integral over $r$ (which can be performed by a change of variables) we obtain a function $\left|A\left(\mathbf{x}-\mathbf{x}^{\prime}, \mathbf{b}\right)\right|^{-\frac{1}{2-\alpha-\gamma}}$ (where $\mathbf{b}$ depends on $\sigma$ and $\sigma^{\prime}$ ) whose expectation value is expressed by the rhs of eq.(37). This is an integrable function of $\mathbf{u}, \mathbf{w}, \mathbf{z}, \mathbf{u}^{\prime}, \mathbf{w}^{\prime}$ and $\mathbf{z}^{\prime}$. Hence, it can be bounded by a constant $c_{2}$. Under a stronger assumption that

$$
\int d \mathbf{x} m_{1}(\mathbf{x})<\infty
$$

from eq.(37) we obtain in a similar way the bound

$$
\begin{aligned}
& \int d \mathbf{x}\left\langle\tilde{T}_{\infty}(\mathbf{x}, \mathbf{p}) \tilde{T}_{\infty}\left(\mathbf{x}^{\prime}, \mathbf{p}^{\prime}\right)\right\rangle \\
& \leq \delta\left(\mathbf{p}+\mathbf{p}^{\prime}\right)\left|\tilde{m}_{0}\right|(\mathbf{p})\left(c_{3} \theta\left(|\mathbf{p}|-\frac{1}{\mu}\right)|\mathbf{p}|^{-2}+c_{4} \theta\left(\frac{1}{\mu}-|\mathbf{p}|\right)|\mathbf{p}|^{-\frac{2}{2-\alpha-\gamma}}\right)
\end{aligned}
$$

This is a bound on the spectral measure on the rhs of eq.(7).

We wish to estimate the dependence of the correlation functions (34) on $\mathbf{x}-\mathbf{x}^{\prime}$ in a more explicit form. Note that if the velocity correlations are defined by eq.(9) where $\tilde{D}(\mathbf{k})$ is an integrable function then on the basis of the Lebesgue lemma $G$ is vanishing at large $\left|\mathbf{x}-\mathbf{x}^{\prime}\right|$. In such a case the term depending on 
$\mathbf{x}-\mathbf{x}^{\prime}$ in the exponential on the rhs of eq.(34) can be neglected. If $m$ is in addition a slowly varying function of $\mathbf{x}-\mathbf{x}^{\prime}$ then

$$
\left\langle\tilde{T}_{\tau}(\mathbf{x}, \mathbf{p}) \tilde{T}_{\tau}\left(\mathbf{x}^{\prime}, \mathbf{p}^{\prime}\right)\right\rangle \simeq\left\langle\tilde{T}_{\tau}(\mathbf{x}, \mathbf{p}) \tilde{T}_{\tau}\left(\mathbf{x}, \mathbf{p}^{\prime}\right)\right\rangle
$$

There remains to discuss the turbulent flow (10). We are unable to prove precise upper bounds for large $\left|\mathbf{x}-\mathbf{x}^{\prime}\right|$ and general $\beta$. However, if $0<2 \beta<1$ and $d=1$ then $g(\mathbf{x})=-|\mathbf{x}|^{2 \beta}$ is a convex function [26]

$$
g\left(\frac{1}{2}(\mathbf{x}+\mathbf{y})\right) \leq \frac{1}{2} g(\mathbf{x})+\frac{1}{2} g(\mathbf{y})
$$

As a consequence

$$
\begin{aligned}
& \exp \left(-r^{2-\alpha+\beta} \mathbf{p}^{2} \Gamma\left(\sigma-\sigma^{\prime}\right)\left|r^{-\frac{1}{2}}\left(\mathbf{x}-\mathbf{x}^{\prime}\right)+\mu \mathbf{b}(\sigma)-\mu \mathbf{b}^{\prime}\left(\sigma^{\prime}\right)\right|^{2 \beta}\right) \\
& \leq \exp \left(-\frac{1}{2} r^{2-\alpha+\beta} \mathbf{p}^{2} \Gamma\left(\sigma-\sigma^{\prime}\right)\left|2 r^{-\frac{1}{2}}\left(\mathbf{x}-\mathbf{x}^{\prime}\right)\right|^{2 \beta}-\frac{1}{2}\left|2 \mu \mathbf{b}(\sigma)-2 \mu \mathbf{b}^{\prime}\left(\sigma^{\prime}\right)\right|^{2 \beta}\right)
\end{aligned}
$$

Hence, under the assumption (31) (after the $r$-integration) the inequalities (36)(37) at $\tau=\infty$ for $0<2 \beta<1$ read

$$
\begin{aligned}
& \left\langle\tilde{T}_{\infty}(\mathbf{x}, \mathbf{p}) \tilde{T}_{\infty}\left(\mathbf{x}^{\prime}, \mathbf{p}^{\prime}\right)\right\rangle \\
& \leq K|\mathbf{p}|^{-\frac{2}{2-\alpha}}\left|\tilde{m}_{0}\right|(\mathbf{p})\left|\mathbf{x}-\mathbf{x}^{\prime}\right|^{-\frac{2 \beta}{2-\alpha}}
\end{aligned}
$$

We expect the inequality (44) to hold true in general (under the assumption (31))for large $\left|\mathbf{x}-\mathbf{x}^{\prime}\right|$ because we obtain such a behavior of the two-point function if in a formal way we take the limit $\left|\mathbf{x}-\mathbf{x}^{\prime}\right| \rightarrow \infty$ in eq.(34) neglecting terms of order $\left|\mathbf{x}-\mathbf{x}^{\prime}\right|^{-1}$.

We discuss now the Jensen inequality (38) for the lower bound. It is sufficient to calculate the expectation value in the exponential (38). First, in the Kraichnan model (35) for the term $-W$ in the exponential appearing in eq.(38) we obtain

$$
\begin{aligned}
& \exp \left(-W\left(\mathbf{x}-\mathbf{x}^{\prime}\right)\right)=\exp \left(-r^{1-\gamma} \int d \mathbf{k p} \tilde{D}(\mathbf{k}) \mathbf{p}\right. \\
& \left.\left(1-\mu^{-2} \mathbf{k}^{-2} \exp \left(i \mathbf{k} r^{-\frac{1}{2}}\left(\mathbf{x}-\mathbf{x}^{\prime}\right)\right)\left(1-\exp \left(-\mu^{2} \mathbf{k}^{2}\right)\right)\right)\right)
\end{aligned}
$$


It is easy to see that

$$
\begin{aligned}
& \exp (-W(\mathbf{0}))=\exp \left(-r^{1-\gamma} \int d \mathbf{k p} \tilde{D}(\mathbf{k}) \mathbf{p}\right. \\
& \left.\left(1-\mu^{-2} \mathbf{k}^{-2}\left(1-\exp \left(-\mu^{2} \mathbf{k}^{2}\right)\right)\right)\right) \geq \exp \left(-c r^{1-\gamma} \mathbf{p}^{2}\right)
\end{aligned}
$$

under the assumptions that $\mathbf{p} \tilde{D} \mathbf{p} \geq|\tilde{D}| \mathbf{p}^{2}, \int d \mathbf{k}|\tilde{D}|(\mathbf{k}) \theta\left(|\mathbf{k}|-\frac{1}{\mu}\right)<\infty$ and

$$
\int d \mathbf{k}|\tilde{D}|(\mathbf{k}) \mathbf{k}^{2} \theta\left(\frac{1}{\mu}-|\mathbf{k}|\right)<\infty
$$

In such a case we can take the limit $\tau \rightarrow \infty$. In this limit

$$
\begin{aligned}
& \left\langle\tilde{T}_{\infty}(\mathbf{x}, \mathbf{p}) \tilde{T}_{\infty}\left(\mathbf{x}, \mathbf{p}^{\prime}\right)\right\rangle \geq \delta\left(\mathbf{p}+\mathbf{p}^{\prime}\right) \tilde{m}_{0}(\mathbf{p}) \int_{0}^{\infty} d r \int d a \nu_{1}(a) \\
& \exp \left(-\mu^{2} \mathbf{p}^{2} r-c \mathbf{p}^{2} r^{1-\gamma}-2 a \mu^{2} r\right) \\
& =\tilde{m}_{0}(\mathbf{p}) \delta\left(\mathbf{p}+\mathbf{p}^{\prime}\right) \int_{0}^{\infty} d r m_{1}(\mu \sqrt{2 r}) \exp \left(-\mu^{2} \mathbf{p}^{2} r-c \mathbf{p}^{2} r^{1-\gamma}\right)
\end{aligned}
$$

The behavior of the integral (47) depends on the behavior of the source correlations $m_{1}$ as a function of $\left|\mathbf{x}-\mathbf{x}^{\prime}\right|$. If

$$
m_{1}(\mu \sqrt{2 r}) \geq K
$$

then

$$
\left\langle\tilde{T}_{\infty}(\mathbf{x}, \mathbf{p}) \tilde{T}_{\infty}\left(\mathbf{x}, \mathbf{p}^{\prime}\right)\right\rangle \geq \delta\left(\mathbf{p}+\mathbf{p}^{\prime}\right) \tilde{m}_{0}(\mathbf{p})\left(c_{5} \theta\left(|\mathbf{p}|-\frac{1}{\mu}\right)|\mathbf{p}|^{-2}+c_{6} \theta\left(\frac{1}{\mu}-|\mathbf{p}|\right)|\mathbf{p}|^{-\frac{2}{1-\gamma}}\right)
$$

This lower bound coincides with the upper bound (40) (where $\alpha=1$ ). If $m_{1}$ satisfies a stronger condition $(\Omega<1)$

$$
m_{1}(|\mathbf{x}|) \geq K|\mathbf{x}|^{-2 \Omega}
$$

( $\Omega \geq 0$ if it is to be of the form (24),i.e., $\nu_{1}(a) \geq K a^{\Omega-1}$ ) then

$$
\left\langle\tilde{T}_{\infty}(\mathbf{x}, \mathbf{p}) \tilde{T}_{\infty}\left(\mathbf{x}, \mathbf{p}^{\prime}\right)\right\rangle \geq \delta\left(\mathbf{p}+\mathbf{p}^{\prime}\right) \tilde{m}_{0}(\mathbf{p})\left(c_{7} \theta\left(|\mathbf{p}|-\frac{1}{\mu}\right)|\mathbf{p}|^{-2+2 \Omega}+c_{8} \theta\left(\frac{1}{\mu}-|\mathbf{p}|\right)|\mathbf{p}|^{-\frac{2-2 \Omega}{1-\gamma}}\right)
$$


The inequality (51) results from the following estimate (for $\alpha+\gamma<1$ )

$$
\begin{aligned}
& \int_{0}^{\infty} d r r^{-\Omega} \exp \left(-\mu^{2} \mathbf{p}^{2} r-c \mathbf{p}^{2} r^{2-\alpha-\gamma}\right) \\
& =\int_{0}^{1} d r r^{-\Omega} \exp \left(-\mu^{2} \mathbf{p}^{2} r-c \mathbf{p}^{2} r^{2-\alpha-\gamma}\right)+\int_{1}^{\infty} d r r^{-\Omega} \exp \left(-\mu^{2} \mathbf{p}^{2} r-c \mathbf{p}^{2} r^{2-\alpha-\gamma}\right) \\
& \geq \int_{0}^{1} d r r^{-\Omega} \exp \left(-\left(\mu^{2} \mathbf{p}^{2}+c \mathbf{p}^{2}\right) r\right)+\int_{1}^{\infty} d r r^{-\Omega} \exp \left(-\left(\mu^{2} \mathbf{p}^{2}+c \mathbf{p}^{2}\right) r^{2-\alpha-\gamma}\right) \\
& =|\mathbf{p}|^{-2+2 \Omega} \int_{0}^{\mathbf{p}^{2}} t^{-\Omega} \exp \left(-\left(\mu^{2}+c\right) t\right) d t+|\mathbf{p}|^{-\frac{2-2 \Omega}{2-\alpha-\gamma}} \int_{a(\mathbf{p})}^{\infty} t^{-\Omega} \exp \left(-\left(\mu^{2}+c\right) t^{2-\alpha-\gamma}\right) d t
\end{aligned}
$$

where $a(\mathbf{p})=|\mathbf{p}|^{\frac{2}{2-\alpha-\gamma}}$ and $\alpha=1$ in application to eq.(47).

Next, we wish to estimate the behavior of the temperature correlations at large $\mathbf{x}-\mathbf{x}^{\prime}$ in the turbulent case (10) when $\gamma=-\beta<0$ (if $\gamma>0$ and $m_{1}$ is a bounded function then the temperature correlations are bounded from below and from above as functions of $\mathbf{x}-\mathbf{x}^{\prime}$,eq.(43)). First, we consider the Kraichnan model $(35)\left(\Gamma\left(s-s^{\prime}\right)=\delta\left(s-s^{\prime}\right)\right.$ in eq.(10)) with the mean velocity $\mathbf{U}=0$ and

$$
\tilde{D}(\mathbf{k}) \simeq|\mathbf{k}|^{-d+2 \gamma}
$$

The integral in eq.(45) is convergent for large $\mathbf{k}$ if $\gamma<0$ and for small $\mathbf{k}$ if $-\gamma<1$. We consider the model (10) with $0<\beta=-\gamma<1$. Let us change the integration variable in eq.(45)

$$
\mathbf{k}=\left|\mathbf{x}-\mathbf{x}^{\prime}\right|^{-1} \sqrt{r} \mathbf{q}
$$

Then, after an estimate of the remainder

$$
\exp \left(-W\left(\mathbf{x}-\mathbf{x}^{\prime}\right)\right) \geq \exp \left(-c r \mathbf{p}^{2}\left|\mathbf{x}-\mathbf{x}^{\prime}\right|^{2 \beta}\right)
$$

As a consequence

$$
\begin{aligned}
& \left\langle\tilde{T}_{\tau}(\mathbf{x}, \mathbf{p}) \tilde{T}_{\tau}\left(\mathbf{x}^{\prime}, \mathbf{p}^{\prime}\right)\right\rangle \\
& \geq \delta\left(\mathbf{p}+\mathbf{p}^{\prime}\right) \tilde{m}_{0}(\mathbf{p})\left(\mu^{2} \mathbf{p}^{2}+c \mathbf{p}^{2}\left|\mathbf{x}-\mathbf{x}^{\prime}\right|^{2 \beta}\right)^{-1}\left(1-\exp \left(-\tau\left(\mu^{2} \mathbf{p}^{2}+c \mathbf{p}^{2}\left|\mathbf{x}-\mathbf{x}^{\prime}\right|^{2 \beta}\right)\right)\right)
\end{aligned}
$$

Hence, for large $\left|\mathbf{x}-\mathbf{x}^{\prime}\right|$ we obtain

$$
\left\langle\tilde{T}_{\infty}(\mathbf{x}, \mathbf{p}) \tilde{T}_{\infty}\left(\mathbf{x}^{\prime}, \mathbf{p}^{\prime}\right)\right\rangle \geq \delta\left(\mathbf{p}+\mathbf{p}^{\prime}\right) \tilde{m}_{0}(\mathbf{p}) c^{-1} \mathbf{p}^{-2}\left|\mathbf{x}-\mathbf{x}^{\prime}\right|^{-2 \beta}
$$


This lower bound for the Kraichnan model is the same as the upper bound (44) (here $\alpha=1$ ).

Let us calculate the expectation value in the exponential of eq.(38) (denoted by $-W$ ) for the general $G$ of eq.(19)

$$
\begin{aligned}
& W\left(\mathbf{x}-\mathbf{x}^{\prime}\right)=r^{2-\alpha-\gamma} \int d \omega d \mathbf{k p} \tilde{G}(\omega, \mathbf{k}) \mathbf{p} \\
& \left(2\left(\frac{1}{2} \mu^{2} \mathbf{k}^{2}-i \omega\right)^{-1}\left(1-\left(\frac{1}{2} \mu^{2} \mathbf{k}^{2}-i \omega\right)^{-1}\left(1-\exp \left(-\frac{1}{2} \mu^{2} \mathbf{k}^{2}+i \omega\right)\right)\right)\right. \\
& \left.-\left(\frac{1}{4} \mu^{4}|\mathbf{k}|^{4}+\omega^{2}\right)^{-1} \exp \left(i \mathbf{k} r^{-\frac{1}{2}}\left(\mathbf{x}-\mathbf{x}^{\prime}\right)\right)\left|1-\exp \left(-\frac{1}{2} \mu^{2} \mathbf{k}^{2}+i \omega\right)\right|^{2}\right)
\end{aligned}
$$

We estimate this integral at $\mathbf{x}=\mathbf{x}^{\prime}$ first. Similarly as in eq.(46) the scale invariance (21) leads to

$$
W(\mathbf{0}) \geq c r^{2-\alpha-\gamma} \mathbf{p}^{2}
$$

if

$$
\int d \mathbf{k} d \omega \tilde{G}(\omega, \mathbf{k})\left(\frac{1}{2} \mu^{2} \mathbf{k}^{2}-i \omega\right)^{-1} \theta\left(|\mathbf{k}|-\frac{1}{\mu}\right)<\infty
$$

and

$$
\iint d \mathbf{k} d \omega \tilde{G}(\omega, \mathbf{k})\left(\frac{1}{4} \mu^{4}|\mathbf{k}|^{4}+\omega^{2}\right)^{\frac{1}{2}} \theta\left(\frac{1}{\mu}-|\mathbf{k}|\right)<\infty
$$

Hence, under the assumption (50) on the basis of the inequalities (52) and (58) we have the lower bound ( generalizing that of eq.(51) to $\alpha \neq 1$ )

$$
\left\langle\tilde{T}_{\infty}(\mathbf{x}, \mathbf{p}) \tilde{T}_{\infty}\left(\mathbf{x}, \mathbf{p}^{\prime}\right)\right\rangle \geq \delta\left(\mathbf{p}+\mathbf{p}^{\prime}\right) \tilde{m}_{0}(\mathbf{p})\left(c \theta\left(|\mathbf{p}|-\frac{1}{\mu}\right)|\mathbf{p}|^{-2+2 \Omega}+c^{\prime} \theta\left(\frac{1}{\mu}-|\mathbf{p}|\right)|\mathbf{p}|^{-\frac{2-2 \Omega}{2-\alpha-\gamma}}\right)
$$

(at $\Omega=0$ this lower bound coincides with the upper bound (40)). Next, if $\left|\mathbf{x}-\mathbf{x}^{\prime}\right|$ is large then for $0<-\gamma=\beta<1$ we obtain from eqs.(21) and (57) the lower bound

$$
\exp \left(-W\left(\mathbf{x}-\mathbf{x}^{\prime}\right)\right) \geq \exp \left(-c \mathbf{p}^{2} r^{2-\alpha}\left|\mathbf{x}-\mathbf{x}^{\prime}\right|^{2 \beta}\right)
$$

where the form of the rhs comes from a change of variables $\mathbf{k}=\mathbf{k}^{\prime}\left|\mathbf{x}-\mathbf{x}^{\prime}\right|^{-1}$ and $\omega=\omega^{\prime}\left|\mathbf{x}-\mathbf{x}^{\prime}\right|^{-2}$ and an estimate of the remainder in eq.(57). 
If we restrict ourselves to $G$ of the form (10) and $2 \beta \geq 1$ then we can derive a more precise lower bound for $\exp (-W)$ with an application of the Hölder inequality

$$
|\mathbf{x}+\mathbf{y}|^{2 \beta} \leq 2^{2 \beta-1}\left(|\mathbf{x}|^{2 \beta}+|\mathbf{y}|^{2 \beta}\right)
$$

From eq.(38) and the Hölder inequality we obtain after an elementary calculation of the expectation value over the Brownian paths

$$
\exp \left(-W\left(\mathbf{x}-\mathbf{x}^{\prime}\right)\right) \geq \exp \left(-C r^{2-\alpha-\gamma} \mathbf{p}^{2}-c r^{2-\alpha}\left|\mathbf{x}-\mathbf{x}^{\prime}\right|^{2 \beta} \mathbf{p}^{2}\right)
$$

Hence, after a calculation of the expectation value in the exponential in eq.(38) the remaining $r$ and the $a$ integrals (from the representation (24)) in the correlation function (38) read

$$
\begin{aligned}
& \int_{0}^{\infty} d r \int d a \nu_{1}(a) \exp \left(-W-a\left|\mathbf{x}-\mathbf{x}^{\prime}\right|^{2}-2 \mu^{2} r a\right) \\
& \geq \frac{1}{2} \int_{0}^{\infty} d r \int d a \nu_{1}(a) \exp \left(-W-a\left|\mathbf{x}-\mathbf{x}^{\prime}\right|^{2}\right)+\frac{1}{2} \int_{0}^{\infty} d r \int d a \nu_{1}(a) \exp \left(-W-2 \mu^{2} r a\right)
\end{aligned}
$$

where $\exp (-W)$ is lower bounded by eq.(60). An easy estimate of this integral leads to the following inequality large $\mathbf{x}-\mathbf{x}^{\prime}$

$$
\begin{aligned}
& \left\langle\tilde{T}_{\infty}(\mathbf{x}, \mathbf{p}) \tilde{T}_{\infty}\left(\mathbf{x}^{\prime}, \mathbf{p}^{\prime}\right)\right\rangle \\
& \geq\left(\delta\left(\mathbf{p}+\mathbf{p}^{\prime}\right) \tilde{m}_{0}(\mathbf{p})\left(K_{1}|\mathbf{p}|^{-\frac{2-2 \Omega}{2-\alpha}}\left|\mathbf{x}-\mathbf{x}^{\prime}\right|^{-2 \sigma}+K_{2}|\mathbf{p}|^{-\frac{2}{2-\alpha}}\left|\mathbf{x}-\mathbf{x}^{\prime}\right|^{-\frac{2 \beta}{2-\alpha}-2 \Omega}\right)\right.
\end{aligned}
$$

where

$$
\sigma=\frac{\beta(1-\Omega)}{2-\alpha}
$$

For $0<2 \beta<1$ this lower bound coincides with the upper bound (44)(derived for $\Omega=0$ ). We expect that eq.(61) gives the asymptotic behavior of the twopoint correlation function for any $0<2 \beta<2$ because such a behavior is a consequence of a formal exchange of the limit $\left|\mathbf{x}-\mathbf{x}^{\prime}\right| \rightarrow \infty$ with the integral over $t$ and the expectation value over the Brownian motion in eq.(34). 
The lower bound (59) for small $\mathbf{p}$ is obtained by neglecting the $\left|\mathbf{x}-\mathbf{x}^{\prime}\right|$ dependent term on the rhs of eq.(60). We can see from eq.(59) that if $\tilde{m}_{0}(\mathbf{p}) \simeq$ $|\mathbf{p}|^{-\nu}$ and $m_{1}(\mathbf{k}) \simeq|\mathbf{k}|^{-d+2 \Omega}$ then the $\langle\tilde{T} \tilde{T}\rangle$ correlations behave as $|\mathbf{p}|^{-2-\nu+2 \Omega}$ for large momenta (short distances in the $\mathbf{z}$ direction), whereas the low momentum behaviour (large distance ) is $|\mathbf{p}|^{-\nu-\frac{2-2 \Omega}{2-\alpha-\gamma}}$. These estimates show the effect of the random flow on the temperature correlations in the $\mathbf{z}$ direction. The effect on the temperature correlations in the $\mathbf{x}$ direction is described by the lower bound (61) and the upper bound (44). Again the decay of temperature correlations is determined by scaling indices of the velocity and source correlations.

\section{Higher order correlation functions}

Let us consider the multi-point correlation functions

$$
\begin{aligned}
& \left\langle\tilde{T}_{\tau}\left(\mathbf{x}_{1}, \mathbf{p}_{1}\right) \ldots \ldots \tilde{T}_{\tau}\left(\mathbf{x}_{2 n}, \mathbf{p}_{2 n}\right)\right\rangle=\sum_{\text {pairs }} \int_{0}^{\tau} d t_{1} \ldots . d t_{2 n} \\
& \prod_{(j, k)} \delta\left(\mathbf{p}_{j}+\mathbf{p}_{k}\right) \delta\left(t_{j}-t_{k}\right) \exp \left(-\frac{1}{2} \mu^{2} \sum_{j} \mathbf{p}_{j}^{2}\left(\tau-t_{j}\right)\right) \\
& E\left[\prod_{(j, k)} \tilde{m}\left(\mathbf{x}_{j}-\mathbf{x}_{k}+\mu \mathbf{b}_{j}\left(\tau-t_{j}\right)-\mu \mathbf{b}_{k}\left(\tau-t_{k}\right), \mathbf{p}_{j}\right)\right. \\
& \left.\exp \left(-\frac{1}{2} \sum_{i l} \int_{0}^{\tau-t_{i}} \int_{0}^{\tau-t_{l}} d s d s^{\prime} \mathbf{p}_{i} G_{0}\left(s-s^{\prime}, \mathbf{x}_{i}-\mathbf{x}_{l}+\mu \mathbf{b}_{i}(s)-\mu \mathbf{b}_{l}\left(s^{\prime}\right)\right) \mathbf{p}_{l}\right)\right]
\end{aligned}
$$

where the sum is over all pairings in accordance with the Gaussian combinatorics. From (63) we have

$$
\begin{aligned}
& \left|\left\langle\tilde{T}_{\tau}\left(\mathbf{x}_{1}, \mathbf{p}_{1}\right) \ldots \ldots \tilde{T}_{\tau}\left(\mathbf{x}_{2 n}, \mathbf{p}_{2 n}\right)\right\rangle\right| \leq \sum_{\text {pairs }} \int_{0}^{\tau} d t_{1} \ldots . d t_{2 n} \\
& \prod_{(j, k)} \delta\left(\mathbf{p}_{j}+\mathbf{p}_{k}\right) \delta\left(t_{j}-t_{k}\right) \exp \left(-\frac{1}{2} \mu^{2} \sum_{j} \mathbf{p}_{j}^{2}\left(\tau-t_{j}\right)\right) \\
& E\left[\prod_{(j, k)}\left|\tilde{m}\left(\mathbf{x}_{j}-\mathbf{x}_{k}+\mu \mathbf{b}_{j}\left(\tau-t_{j}\right)-\mu \mathbf{b}_{k}\left(\tau-t_{k}\right), \mathbf{p}_{j}\right)\right|\right]<\infty
\end{aligned}
$$

Hence, the equilibrium limit $\tau \rightarrow \infty$ exists.

If $m$ is either of the form (24) or (25) then we can apply the Jensen inequality 
to the expectation value in the form $E[\exp f] \geq \exp E[f]$. We obtain an analogue of the lower bound (38). For the upper bound we apply the Jensen inequality to the time integral

$$
\begin{aligned}
& \exp \left(-\frac{1}{2} \int_{0}^{\tau} \int_{0}^{\tau} d s d s^{\prime} \iint J_{k}(s) J_{l}\left(s^{\prime}\right)\left\langle v_{k}(s) v_{l}\left(s^{\prime}\right)\right\rangle\right) \\
& \leq \tau^{-2} \int_{0}^{\tau} \int_{0}^{\tau} d s d s^{\prime} \exp \left(-\frac{\tau^{2}}{2} \iint J_{k}(s) J_{l}\left(s^{\prime}\right)\left\langle v_{k}(s) v_{l}\left(s^{\prime}\right)\right\rangle\right)
\end{aligned}
$$

where

$$
\mathbf{J}(s, \mathbf{u})=-\theta(s) \sum_{k=1}^{2 n} \mathbf{p}_{k} \delta\left(\mathbf{u}-\mathbf{x}_{k}-\mu \mathbf{b}_{k}(\tau-s)\right)
$$

and the additional integral in eq.(64) is over the spatial variable $\mathbf{u}$.

We can repeat the basic estimates concerning the behavior for low $\mathbf{z}$ momenta and large $\mathbf{x}$ distances by means of the methods applied for the two-point correlations. First, by means of the Jensen inequalities we reduce the estimates of the expectation values to finite dimensional integrals. From the Jensen inequalities we can see that the correlation functions are bounded in $\tau$ when $\tau \rightarrow \infty$. Next, the results concerning the scaling behavior for $2 n$-point functions can be obtained by an introduction of spherical coordinates in the $d t_{1} \ldots d t_{n}$ integral in eq.(63). Then, the correlation functions scale in a simple way with respect to the temporal radius $r$. Let us explain such estimates in more detail for $n=2$. Then,

$$
\begin{aligned}
& \left\langle\tilde{T}_{\tau}\left(\mathbf{x}_{1}, \mathbf{p}_{1}\right) \ldots . . \tilde{T}_{\tau}\left(\mathbf{x}_{4}, \mathbf{p}_{4}\right)\right\rangle=\delta\left(\mathbf{p}_{1}+\mathbf{p}_{3}\right) \delta\left(\mathbf{p}_{2}+\mathbf{p}_{4}\right) \\
& \int_{0}^{\tau} d t_{1} \int_{0}^{\tau} d t_{2} \exp \left(-\mu^{2} \mathbf{p}_{1}^{2}\left(\tau-t_{1}\right)-\mu^{2} \mathbf{p}_{2}^{2}\left(\tau-t_{2}\right)\right) \\
& E\left[\tilde{m}\left(\mathbf{x}_{1}-\mathbf{x}_{3}+\mu \mathbf{b}_{1}\left(\tau-t_{1}\right)-\mu \mathbf{b}_{3}\left(\tau-t_{1}\right), \mathbf{p}_{1}\right)\right. \\
& \tilde{m}\left(\mathbf{x}_{2}-\mathbf{x}_{4}+\mu \mathbf{b}_{2}\left(\tau-t_{2}\right)-\mu \mathbf{b}_{4}\left(\tau-t_{2}\right), \mathbf{p}_{2}\right) \\
& \exp \left(-\sum_{j=1,2} \int_{0}^{\tau-t_{j}} \int_{0}^{\tau-t_{j}} d t d t^{\prime} \mathbf{p}_{j} G_{0}\left(t-t^{\prime}, \mu \mathbf{b}_{j}(t)-\mu \mathbf{b}_{j}\left(t^{\prime}\right)\right) \mathbf{p}_{j}\right. \\
& \left.\left.+\sum_{j<k} \int_{0}^{\tau-t_{j}} \int_{0}^{\tau-t_{k}} d t d t^{\prime} \mathbf{p}_{j} G_{0}\left(t-t^{\prime}, \mathbf{x}_{j}-\mathbf{x}_{k}+\mu \mathbf{b}_{j}(t)-\mu \mathbf{b}_{k}\left(t^{\prime}\right)\right) \mathbf{p}_{k}\right)\right]+ \text { permut } .
\end{aligned}
$$


where the sum is over permutations of the numbers from 1 to 4 in accordance with the Gaussian combinatorics; in the sum in the exponential we set $t_{1}=t_{3}$ and $t_{2}=t_{4}$. Let $\tau-t_{1}=r \cos \theta, \tau-t_{2}=r \sin \theta, t=r \sigma \cos \theta$ and $t^{\prime}=$ $r \sigma^{\prime} \sin \theta$. In such a case $r$ scales in the exponential in the same way as in eqs.(37)-(38). The integral $d t_{1} d t_{2}=d r r d \theta$ adds an additional power of $\mathrm{r}$. Under the assumption (31) the small $\mathbf{p}$ behavior of the correlation functions (65) at $\tau=\infty$ is determined by the integral

$$
\begin{aligned}
& \left|\left\langle\tilde{T}_{\infty}\left(\mathbf{x}_{1}, \mathbf{p}_{1}\right) \ldots \ldots . \tilde{T}_{\infty}\left(\mathbf{x}_{4}, \mathbf{p}_{4}\right)\right\rangle\right| \\
& \simeq\left|\tilde{m}_{0}\right|\left(\mathbf{p}_{1}\right)\left|\tilde{m}_{0}\right|\left(\mathbf{p}_{2}\right) \int_{0}^{\infty} d r r E\left[\exp \left(-r^{2-\alpha-\gamma} \sum_{j k} \mathbf{p}_{j} G_{0} \mathbf{p}_{k}\right)\right]+\text { permut. } \\
& \simeq\left|\tilde{m}_{0}\right|\left(\mathbf{p}_{1}\right)\left|\tilde{m}_{0}\right|\left(\mathbf{p}_{2}\right) E\left[\left|\sum_{j k} \mathbf{p}_{j} G_{0} \mathbf{p}_{k}\right|^{-\frac{2}{2-\alpha-\gamma}}\right]+\text { permut. }
\end{aligned}
$$

For large distances , $\gamma=-\beta<0$ and $G$ of eq.(10) we can expand the dependence on the Brownian motion in eq.(66) in powers of $\mu\left|\mathbf{x}_{j}-\mathbf{x}_{k}\right|^{-1}$. The leading order reads

$$
\begin{aligned}
& \left|\left\langle\tilde{T}_{\infty}\left(\mathbf{x}_{1}, \mathbf{p}_{1}\right) \ldots \ldots . \tilde{T}_{\infty}\left(\mathbf{x}_{4}, \mathbf{p}_{4}\right)\right\rangle\right| \\
& \simeq\left|\tilde{m}_{0}\right|\left(\mathbf{p}_{1}\right)\left|\tilde{m}_{0}\right|\left(\mathbf{p}_{2}\right)\left|\sum_{j k} \mathbf{p}_{j} \mathbf{p}_{k}\right| \mathbf{x}_{j}-\left.\left.\mathbf{x}_{k}\right|^{2 \beta}\right|^{-\frac{2}{2-\alpha}}+\text { permut } .
\end{aligned}
$$

Note that the power describing the low $\mathbf{p}$ behavior in eq.(66) and large $\mathbf{x}$ behavior in eq.(67) is twice as big as that for the two-point function (34) and (40) indicating the asymptotic scale invariance of the temperature $\tilde{T}_{\infty}(\mathbf{x}, \mathbf{p})$ at low momenta or large distances. This property can be extended to the $2 n$ correlation functions where the scaling index is proportional to $n$ as a consequence of the $d r r^{n-1}$ time integral in the spherical time coordinates. Such a behavior of the integrals suggests that if the velocities and the sources are scale invariant then the temperatures scale at large distances with the scale dimension determined by the two-point function. 


\section{Discussion}

The power-law behavior of turbulent velocity correlation functions and passive scalar correlation functions in a homogeneous isotropic turbulent flow has been widely discussed in the literature since the basic papers of Kolmogorov [2] followed by Obukhov [28],Corrsin[29] and Batchelor[30] (concerning the scalar

advection). The universal Kolmogorov $\frac{5}{3}$ law for spectral velocity distribution as well as passive scalar distribution is derived by means of dimensional arguments (independent of any dynamical model). A statistical homogeneity and isotropy of the turbulence at a microscale in a sufficiently large space interval (called the " inertial range") is at the base of the Kolmogorov theory. Under these assumptions the velocity (or passive scalar) correlation functions are universal ,i.e.,independent of the source distribution $m$. An experimental verification is not simple. Turbulent flows are usually non-homogeneous and non-isotropic at a macro scale. However, if a flow satisfying Kolmogorov assumptions is created then the spectral Kolmogorov law is satisfied in the inertial range [31]. Nevertheless, it is common for flows in nature that Kolmogorov assumptions are not satisfied (for some studies of such turbulent flows see [32][33]). Even if the velocity is satisfying the Kolmogorov law the analogous Obukhov law for $\rho$ may fail [32][33][34]. As the authors in [32] point out some problems with the verification of Kolmogorov's theory concerns a construction of a flow which would be homogeneous and isotropic in a sufficiently large inertial range ( usually boundary conditions or sources violate a global symmetry). They suggest a study of non-isotropic flows.

An investigation of a general class of dynamical models of randomly forced Navier-Stokes and passive scalar equations is still beyond the reach of analytical 
as well as numerical methods. A substantial progress has been achieved in the white noise randomly forced passive scalar (Kraichnan model)[8] [4][9]. However, the white noise distribution of velocities is quite unrealistic. Our main motivation in these studies was a derivation of the scaling behavior for velocities which are not of the white noise type. A passive scalar in a shear flow independent of the coordinates in the direction of the flow was studied before in $[6][7]$. However, these authors were interested in the anomalous free decay of solutions of the advection-diffusion equation.

Our results predict a power-law of the passive scalar correlations in nonisotropic flows. The results depend on the source distribution $m$ because the source $f$ is present at any scale. We do not specify any inertial range in our model. In general, the correlations must depend on the source (for a discussion of random forcing see [35]). This can be seen from the detailed calculations in [36][37] performed in the isotropic Kraichnan model (white noise in time) [8][4] [9]. The two-point passive scalar correlations depend explicitly on the source and on the molecular diffusivity $\mu^{2}$. Only in a proper limit of the source covariance $m$ and $\mu \rightarrow 0$ the universal scaling law comes out.

Before we summarize our results let us begin with simple models. First, consider a pure diffusion corresponding to $\mathbf{V}=0$. Then

$$
\begin{aligned}
& \left\langle\tilde{T}_{\tau}(\mathbf{x}, \mathbf{p}) \tilde{T}_{\tau}\left(\mathbf{x}^{\prime}, \mathbf{p}^{\prime}\right)\right\rangle=2 \delta\left(\mathbf{p}+\mathbf{p}^{\prime}\right) \int_{0}^{\tau} d r \exp \left(-\mu^{2} \mathbf{p}^{2} r\right) \\
& E\left[\tilde{m}\left(\mathbf{x}-\mathbf{x}^{\prime}+\mu \sqrt{r} \mathbf{b}(1)-\mu \sqrt{r} \mathbf{b}^{\prime}(1), \mathbf{p}\right)\right] \\
& =2 \delta\left(\mathbf{p}+\mathbf{p}^{\prime}\right)(2 \pi)^{-D+d} \int_{0}^{\tau} d r \exp \left(-\mu^{2} \mathbf{p}^{2} r\right) \\
& \left.\int d \mathbf{u} d \mathbf{w} \exp \left(-\frac{\mathbf{u}^{2}}{2}-\frac{\mathbf{w}^{2}}{2}\right) \tilde{m}\left(\mathbf{x}-\mathbf{x}^{\prime}+\mu \sqrt{r} \mathbf{u}-\mu \sqrt{r} \mathbf{w}, \mathbf{p}\right)\right] \\
& =\mu^{-2} \delta\left(\mathbf{p}+\mathbf{p}^{\prime}\right) \int d \mathbf{k} \exp \left(i \mathbf{k}\left(\mathbf{x}-\mathbf{x}^{\prime}\right)\right) \tilde{m}_{1}(\mathbf{k}) \tilde{m}_{0}(\mathbf{p})\left(\mathbf{p}^{2}+\mathbf{k}^{2}\right)^{-1} \\
& \left(1-\exp \left(-\mu^{2}\left(\mathbf{p}^{2}+\mathbf{k}^{2}\right) \tau\right)\right)
\end{aligned}
$$


In the limit $\tau \rightarrow \infty$

$$
\left\langle T_{\infty}(\mathbf{x}, \mathbf{z}) T_{\infty}\left(\mathbf{x}^{\prime}, \mathbf{z}^{\prime}\right)\right\rangle=\mu^{-2} \int d \mathbf{k} d \mathbf{p} \exp \left(i \mathbf{k}\left(\mathbf{x}-\mathbf{x}^{\prime}\right)\right) \exp \left(i \mathbf{p}\left(\mathbf{z}-\mathbf{z}^{\prime}\right)\right) \tilde{m}(\mathbf{k}, \mathbf{p})\left(\mathbf{p}^{2}+\mathbf{k}^{2}\right)^{-1}
$$

Hence

$$
\rho_{\infty}(\mathbf{k}, \mathbf{p})=\mu^{-2}\left(\mathbf{p}^{2}+\mathbf{k}^{2}\right)^{-1} \tilde{m}_{1}(\mathbf{k}) \tilde{m}_{0}(\mathbf{p})
$$

Let us note that the behavior of the temperature correlations changes abruptly for large $\left|\mathbf{z}-\mathbf{z}^{\prime}\right|$ at $\tau=\infty$ in this simple model. At finite $\tau$ it is the same as that of the source (say $\left|\mathbf{z}-\mathbf{z}^{\prime}\right|^{-d+\nu}$ ) whereas at $\tau=\infty$ it becomes $\mid \mathbf{z}-$ $\left.\mathbf{z}^{\prime}\right|^{-d+\nu+2}$. However, it can be seen from eq.(68) that after the limit $\tau \rightarrow \infty$ the limit $\mu \rightarrow 0$ does not exist in the model without the advection. If we first take $\mu \rightarrow 0$ then the subsequent limit $\tau \rightarrow \infty$ is linearly divergent in $\tau$. The strong $\mu$-dependence of the asymptotic behavior means that this parameter sets a scale on time and space which determines different scaling behavior. In Appendix A we show that the limits $\mu \rightarrow 0$ and $\tau \rightarrow \infty$ can be interchanged in the model with a random advection. The correlation functions $\mathcal{S}^{(2 n)}$ in a non-isotropic Kraichnan model are discussed in Appendix B. The correlation functions $\mathcal{S}^{(2 n)}\left(\mathbf{x}_{1}, \mathbf{p}_{1}, \ldots . ., \mathbf{x}_{2 n}, \mathbf{p}_{2 n}\right)$ can be calculated exactly in the limit $\mu \rightarrow 0$ (eq.(86)). They show no anomalous scaling (encountered in the isotropic model [8][4]) as long as the points $\mathbf{x}_{j}$ are different. The scaling behavior can change after a transformation to the configuration space (the Fourier transform does not exist in the usual sense).

Let us compare the two-point temperature correlation function (68) with the one in a random flow which is bounded in space and time, i.e., $G=\langle\mathbf{v v}\rangle \simeq$ const. Under the assumption (31) we obtain 


$$
\left\langle\tilde{T}_{\infty}(\mathbf{x}, \mathbf{p}) \tilde{T}_{\infty}\left(\mathbf{x}^{\prime}, \mathbf{p}^{\prime}\right)\right\rangle \simeq K \delta\left(\mathbf{p}+\mathbf{p}^{\prime}\right) \tilde{m}_{0}(\mathbf{p}) \int_{0}^{\infty} d r \exp \left(-\mu^{2} \mathbf{p}^{2} r-c \mathbf{p}^{2} r^{2}\right)
$$

The integral (70) behaves as $\tilde{m}_{0}(\mathbf{p}) \mathbf{p}^{-2}$ for large $\mathbf{p}$ and as $\tilde{m}_{0}(\mathbf{p})|\mathbf{p}|^{-1}$ for a small $\mathbf{p}$ in agreement with eq.(59) for $\Omega=\alpha=\gamma=0$. Our results of secs. 4 and 5 give an extension of the simple observations on the temperature correlation functions derived in this section for a pure diffusion and for an advection by a uniformly bounded random flow.

In our model (defined by the assumption that the velocity does not depend on coordinates in the direction of the flow) the spectral distribution in the corresponding momentum is proportional to the source distribution $\tilde{m}$ as can be seen from eq.(34). We could consider a source $f$ with the covariance $m(\mathbf{x}, \mathbf{z})$ which (approximately in a certain range as in refs.[36][37]) is independent of $\mathbf{x}$. In such a case the spectral equilibrium distribution (6) for a pure diffusion $\rho_{\infty}(\mathbf{k}, \mathbf{p})(67)$ is $\delta(\mathbf{k}) \tilde{m}_{0}(\mathbf{p}) \mathbf{p}^{-2}$ where the $\mathbf{p}^{-2}$ behavior comes from the molecular diffusivity. The temperature correlations remain independent of $\mathbf{x}$ and the limit $\mu \rightarrow 0$ does not exist. A random advection is changing the behavior of temperature correlations in $\mathbf{x}$ as well as in $\mathbf{p}$. This change involves a non-perturbative mechanism which could not be seen in an expansion in $\mathbf{V}$. It comes from an exponential of $G$ in eq.(34). In particular, a steady flow bounded in $\mathbf{x}$ gives $\rho_{\infty}(\mathbf{k}, \mathbf{p})=\delta(\mathbf{k})|\mathbf{p}|^{-1}$ for $|\mathbf{p}| \ll \frac{1}{\mu}$ whereas for the random velocity growing in space with the index $\beta$ (eq.(10)) we have for a small $\mathbf{k}$ the behavior $\rho_{\infty}(\mathbf{k}, \mathbf{p}) \simeq|\mathbf{k}|^{-d+\frac{2 \beta}{2-\alpha}}$ as follows from eq.(61).

In experiments $(D=3)$ we could create an anisotropic flow with the Kolmogorov index (10) $\beta=\frac{1}{3}$ in $d=2$ or $d=1$. In such a case we obtain definite predictions concerning the temperature distribution. This will be 
$\left|\mathbf{x}-\mathbf{x}^{\prime}\right|^{-\frac{2}{3}}\left|\mathbf{z}-\mathbf{z}^{\prime}\right|^{\nu}$ where

$$
\nu=\frac{2}{2-\alpha}-(D-d)
$$

and $D-d$ is either 1 or 2 and there is a restriction $\alpha-\beta<1$ coming from the requirement of the integrability of the expression in the exponential of (34).

In general, we can see from eqs.(40),(42),(44),(59) and (61) that the turbulent behavior $\gamma=-\beta<0$ of the velocity field will (in comparison to pure diffusion) decrease the temperature correlations in the direction orthogonal to the flow and increase the correlations (at the fixed $\alpha$ )in the direction of the flow. These effects contribute to the more coherent heat distribution in a turbulent stream.

\section{Appendix A: The limit $\mu \rightarrow 0$}

If there is no diffusion $(\mu=0)$ then our formulas in secs.4-6 at finite $\tau$ remain valid but need some interpretation. There is no expectation value over the Brownian motion. In such a case in some formulas (as in eqs.(34)-(35)) $\gamma=0$. Let us consider as an example the formula (34) at $\mu=0$

$$
\begin{aligned}
& \left\langle\tilde{T}_{\tau}(\mathbf{x}, \mathbf{p}) \tilde{T}_{\tau}\left(\mathbf{x}^{\prime}, \mathbf{p}^{\prime}\right)\right\rangle=\delta\left(\mathbf{p}+\mathbf{p}^{\prime}\right) \tilde{m}\left(\mathbf{x}-\mathbf{x}^{\prime}, \mathbf{p}\right) \int_{0}^{\tau} d t \exp \left(-i \mathbf{p} \int_{0}^{\tau-t} d s \mathbf{U}(\tau-s, \mathbf{x})\right) \\
& \exp \left(-(\tau-t)^{2-\alpha} \int_{0}^{1} \int_{0}^{1} d \sigma d \sigma^{\prime} \mathbf{p} G_{0}\left(\sigma-\sigma^{\prime}, \mathbf{0}\right) \mathbf{p}\right. \\
& \left.+(\tau-t)^{2-\alpha} \int_{0}^{1} \int_{0}^{1} d \sigma d \sigma^{\prime} \mathbf{p} G_{0}\left(\sigma-\sigma^{\prime}, \mathbf{x}-\mathbf{x}^{\prime}\right) \mathbf{p}\right)
\end{aligned}
$$

For the Kraichnan model [8] (35) the formula (71) reads (with the Stratonovitch interpretation of the gradient term, see the discussion at the beginning of sec.2). 


$$
\begin{aligned}
& \left\langle\tilde{T}_{\tau}(\mathbf{x}, \mathbf{p}) \tilde{T}_{\tau}\left(\mathbf{x}^{\prime}, \mathbf{p}^{\prime}\right)\right\rangle=\delta\left(\mathbf{p}+\mathbf{p}^{\prime}\right) \tilde{m}\left(\mathbf{x}-\mathbf{x}^{\prime}, \mathbf{p}\right) \int_{0}^{\tau} d t \exp \left(-i \mathbf{p} \int_{0}^{\tau-t} \mathbf{U}(\tau-s, \mathbf{x}) d s\right) \\
& \exp \left(-(\tau-t) \mathbf{p} D_{0}(\mathbf{0}) \mathbf{p}+(\tau-t) \mathbf{p} D_{0}\left(\mathbf{x}-\mathbf{x}^{\prime}\right) \mathbf{p}\right)
\end{aligned}
$$

In the limit $\tau \rightarrow \infty$ and for $\mathbf{U}=0$ we can calculate the integral over time in eq.(71) with the result

$$
\begin{aligned}
& \left\langle\tilde{T}_{\infty}(\mathbf{x}, \mathbf{p}) \tilde{T}_{\infty}\left(\mathbf{x}^{\prime}, \mathbf{p}^{\prime}\right)\right\rangle=C \delta\left(\mathbf{p}+\mathbf{p}^{\prime}\right) \tilde{m}\left(\mathbf{x}-\mathbf{x}^{\prime}, \mathbf{p}\right) \\
& \left(\int_{0}^{1} \int_{0}^{1} d \sigma d \sigma^{\prime} \mathbf{p} G_{0}\left(\sigma-\sigma^{\prime}, \mathbf{0}\right) \mathbf{p}-\int_{0}^{1} \int_{0}^{1} d \sigma d \sigma^{\prime} \mathbf{p} G_{0}\left(\sigma-\sigma^{\prime}, \mathbf{x}-\mathbf{x}^{\prime}\right) \mathbf{p}\right)^{-\frac{1}{2-\alpha}}
\end{aligned}
$$

in agreement with the bounds (56) and (61). We can also calculate the higher order correlation functions. As an example, the four point function (65) reads

$$
\begin{aligned}
& \left\langle\tilde{T}_{\tau}\left(\mathbf{x}_{1}, \mathbf{p}_{1}\right) \ldots . . . \tilde{T}_{\tau}\left(\mathbf{x}_{4}, \mathbf{p}_{4}\right)\right\rangle \\
& =\delta\left(\mathbf{p}_{2}+\mathbf{p}_{4}\right) \delta\left(\mathbf{p}_{1}+\mathbf{p}_{3}\right) \tilde{m}\left(\mathbf{x}_{1}-\mathbf{x}_{3}, \mathbf{p}_{1}\right) \tilde{m}\left(\mathbf{x}_{2}-\mathbf{x}_{4}, \mathbf{p}_{2}\right) \\
& \int_{0}^{\tau} d t_{1} \int_{0}^{\tau} d t_{2} \exp \left(-\sum_{j=1,2} \int_{0}^{\tau-t_{j}} \int_{0}^{\tau-t_{j}} d t d t^{\prime} \mathbf{p}_{j} G_{0}\left(t-t^{\prime}, \mathbf{0}\right) \mathbf{p}_{j}\right. \\
& \left.+\sum_{j<k} \int_{0}^{\tau-t_{j}} \int_{0}^{\tau-t_{k}} d t d t^{\prime} \mathbf{p}_{j} G_{0}\left(t-t^{\prime}, \mathbf{x}_{j}-\mathbf{x}_{k}\right) \mathbf{p}_{k}\right)+ \text { permut }
\end{aligned}
$$

We can obtain detailed estimates of the time integrals for any $\alpha$. In some special cases the integrals can be explicitly calculated. In Appendix B we give the formula (eq.(88)) for the Kraichnan model $(\alpha=1)$. For a steady flow $(\Gamma(s)=1$ in eq. $(10), \alpha=0)$ at $\tau=\infty$ the integration over $t_{j}$ gives

$$
\begin{aligned}
& \left\langle\tilde{T}_{\infty}\left(\mathbf{x}_{1}, \mathbf{p}_{1}\right) \ldots \ldots \tilde{T}_{\infty}\left(\mathbf{x}_{4}, \mathbf{p}_{4}\right)\right\rangle \\
& =\delta\left(\mathbf{p}_{2}+\mathbf{p}_{4}\right) \delta\left(\mathbf{p}_{1}+\mathbf{p}_{3}\right) \tilde{m}\left(\mathbf{x}_{1}-\mathbf{x}_{3}, \mathbf{p}_{1}\right) \tilde{m}\left(\mathbf{x}_{2}-\mathbf{x}_{4}, \mathbf{p}_{2}\right) \\
& \left(4 \mathbf{p}_{1}^{2} \mathbf{p}_{2}^{2} D_{0}\left(\mathbf{x}_{1}-\mathbf{x}_{3}\right) D_{0}\left(\mathbf{x}_{2}-\mathbf{x}_{4}\right)\right. \\
& \left.-\left(\mathbf{p}_{1} \mathbf{p}_{2}\right)^{2}\left(D_{0}\left(\mathbf{x}_{1}-\mathbf{x}_{4}\right)+D_{0}\left(\mathbf{x}_{2}-\mathbf{x}_{3}\right)+D_{0}\left(\mathbf{x}_{1}-\mathbf{x}_{2}\right)+D_{0}\left(\mathbf{x}_{3}-\mathbf{x}_{4}\right)\right)^{2}\right)^{-\frac{1}{2}}+\text { permut } .
\end{aligned}
$$

where $D_{0}\left(\mathbf{x}_{j}-\mathbf{x}_{k}\right)=-\left|\mathbf{x}_{j}-\mathbf{x}_{k}\right|^{2 \beta}$ in the model (10). 


\section{Appendix B:The Kraichnan model}

If $\Gamma\left(t-t^{\prime}\right)=\delta\left(t-t^{\prime}\right)$ then we obtain a closed set of equations for the correlation functions

$$
\mathcal{S}_{\tau}^{(n)}\left(\mathbf{x}_{1}, \ldots, \mathbf{x}_{n} ; \mathbf{p}_{1}, \ldots, \mathbf{p}_{n}\right)=\left\langle\tilde{T}_{\tau}\left(\mathbf{x}_{1}, \mathbf{p}_{1}\right) \ldots . \tilde{T}_{\tau}\left(\mathbf{x}_{n}, \mathbf{p}_{n}\right)\right\rangle
$$

These equations have been derived by Kraichnan [8] for velocities depending on all coordinates. In our simplified model (9)-(10) they read (the odd order correlation functions are zero)

$$
\begin{aligned}
& \partial_{\tau} \mathcal{S}_{\tau}^{(2 n)}=\frac{1}{2} \mu^{2} \sum_{j=1}^{j=2 n} \triangle_{j} \mathcal{S}_{\tau}^{(2 n)}-\frac{1}{2}\left(\mu^{2}+D_{0}(\mathbf{0})\right) \sum_{j=1}^{j=2 n} \mathbf{p}_{j}^{2} \mathcal{S}_{\tau}^{(2 n)} \\
& +\sum_{<j, k>} \mathbf{p}_{j} D_{0}\left(\mathbf{x}_{j}-\mathbf{x}_{k}\right) \mathbf{p}_{k} \mathcal{S}_{\tau}^{(2 n)}+\sum_{<j, k>} \delta\left(\mathbf{p}_{j}+\mathbf{p}_{k}\right) \tilde{m}\left(\mathbf{x}_{j}-\mathbf{x}_{k}, \mathbf{p}_{j}\right) \mathcal{S}_{\tau}^{(2 n-2)}(j k) \\
& \equiv \mathcal{M} S_{\tau}^{(2 n)}+\mathcal{R} \mathcal{S}_{\tau}^{(2 n-2)}
\end{aligned}
$$

where $D_{0}$ is the translation invariant part of $D$ and $\mathcal{S}(j k)$ means that the coordinates $\mathbf{x}_{j}$ and $\mathbf{x}_{k}$ are lacking in $\mathcal{S}$. The term $D(\mathbf{0})$ (adding to $\mu^{2}$ ) comes from the Stratonovitch interpretation of eq.(1).The solution of eq.(76) reads

$$
\mathcal{S}_{\tau}^{(2 n)}=\exp (\tau \mathcal{M}) S_{0}^{(2 n)}+\int_{0}^{\tau} d t \exp ((\tau-t) \mathcal{M}) \mathcal{R} \mathcal{S}_{t}^{(2 n-2)}
$$

If the operator $\mathcal{M}$ is strictly negative in the space $L^{2}\left(R^{2 d n}\right)$ then the limit $\tau \rightarrow \infty$ exists and does not depend on the initial condition $\mathcal{S}_{0}^{(2 n)}$.

We can express the solution of eq.(76) by means of the Feynman-Kac formula for the heat kernel

$(\exp (r \mathcal{M}) g)\left(\mathbf{x}_{1}, \ldots \ldots, \mathbf{x}_{2 n}\right)=E\left[\exp \left(\int_{0}^{r} d s W(\mathbf{b}(s))\right) g\left(\mathbf{x}_{1}+\mu \mathbf{b}_{1}(r), \ldots, \mathbf{x}_{2 n}+\mu \mathbf{b}_{2 n}(r)\right)\right]$

where

$$
W(s)=-\frac{1}{2}\left(\mu^{2}+D_{0}(\mathbf{0})\right) \sum_{j=1}^{j=2 n} \mathbf{p}_{j}^{2}+\sum_{<j, k>} \mathbf{p}_{j} D_{0}\left(\mathbf{x}_{j}+\mu \mathbf{b}_{j}(s)-\mathbf{x}_{k}-\mu \mathbf{b}_{k}(s)\right) \mathbf{p}_{k}
$$


We obtain an upper bound on the correlation functions (78) from the Jensen inequality as applied to the time integral

$$
(\exp r \mathcal{M}) g)\left(\mathbf{x}_{1}, \ldots . ., \mathbf{x}_{2 n}\right) \leq \frac{1}{r} \int_{0}^{r} d s E\left[\exp (r W(\mathbf{b}(s)))|g|\left(\mathbf{x}_{1}+\mu \mathbf{b}_{1}(r), \ldots, \mathbf{x}_{2 n}+\mu \mathbf{b}_{2 n}(r)\right)\right]
$$

If $g=\exp h$ (or a superposition with positive coefficients of such functions as in eq.(25))then we have the lower bound from the Jensen inequality as applied to the expectation value

$$
\left.(\exp (r \mathcal{M}) \exp h)\left(\mathbf{x}_{1}, \ldots . ., \mathbf{x}_{2 n}\right) \geq \exp E\left[\int_{0}^{r} d s W(\mathbf{b}(s))\right)+h\left(\mathbf{x}_{1}+\mu \mathbf{b}_{1}(r), \ldots, \mathbf{x}_{2 n}+\mu \mathbf{b}_{2 n}(r)\right)\right]
$$

As an example, the formula for the two point function (in the limit $\tau \rightarrow \infty$ ) with the velocity correlations defined by eq.(10) reads

$$
\begin{aligned}
& \mathcal{S}_{\infty}^{(2)}\left(\mathbf{x}_{1}, \mathbf{x}_{2}, \mathbf{p}_{1}, \mathbf{p}_{2}\right)=\delta\left(\mathbf{p}_{1}+\mathbf{p}_{2}\right) \int_{0}^{\infty} d r \exp \left(-r \mu^{2} \mathbf{p}_{1}^{2}\right) \\
& E\left[\exp \left(-\mathbf{p}_{1}^{2} \int_{0}^{r} d s\left|\mathbf{x}_{1}-\mathbf{x}_{2}+\mu \mathbf{b}_{1}(s)-\mu \mathbf{b}_{2}(s)\right|^{2 \beta}\right) \tilde{m}\left(\mathbf{x}_{1}+\mu \mathbf{b}_{1}(r)-\mathbf{x}_{2}-\mu \mathbf{b}_{2}(r), \mathbf{p}_{1}\right)\right]
\end{aligned}
$$

Then, the resulting correlation functions are controlled from below and from above by the Jensen inequalities. For the lower bound (81) we obtain an explicit formula (using the representation (24) for $m_{1}$ )

$$
\begin{aligned}
& \mathcal{S}_{\infty}^{(2)}\left(\mathbf{x}_{1}, \mathbf{x}_{2}, \mathbf{p}_{1}, \mathbf{p}_{2}\right) \geq \delta\left(\mathbf{p}_{1}+\mathbf{p}_{2}\right) \tilde{m}_{0}(\mathbf{p}) \int d \nu_{1}(a) \int_{0}^{\infty} d r \exp \left(-r \mu^{2} \mathbf{p}_{1}^{2}\right) \\
& \exp \left(-\mathbf{p}^{2} r^{\beta+1} h\left(r^{-\frac{1}{2}}\left|\mathbf{x}_{1}-\mathbf{x}_{2}\right|\right)-a\left|\mathbf{x}_{1}-\mathbf{x}_{2}\right|^{2}-2 \mu^{2} r a\right)
\end{aligned}
$$

where

$$
h(\rho)=K \rho^{2(1+\beta)} \int_{0}^{\rho^{-2}} d \lambda \int_{0}^{\infty} d b b^{-1-\beta}\left(1-\left(1+2 \mu^{2} \lambda b\right)^{-\frac{d}{2}} \exp \left(-\frac{b}{2\left(1+2 \mu^{2} b \lambda\right)}\right)\right)
$$

here $K$ is a positive constant. From eq.(84) it can easily be seen that for large 
$\mathbf{x}-\mathbf{x}^{\prime}$ (small $\rho$ in eq.(84)) the $r$-integrand in eq.(83) behaves as

$$
\exp \left(-K r \mathbf{p}^{2}\left|\mathbf{x}_{1}-\mathbf{x}_{2}\right|^{2 \beta}-a\left|\mathbf{x}_{1}-\mathbf{x}_{2}\right|^{2}-2 \mu^{2} r a\right)
$$

(as shown in another way in eq.(60);here $\alpha=1$ )leading as a consequence to the estimate (61) for the correlation functions. We can continue the Jensen inequalities for higher correlation functions as from eqs.(77) and (78) it follows that the correlation functions are again in the form of superpositions of exponentials.

For lower order correlations a direct study of the differential equation (76) can be equally efficient. As an example, if $D=3$ and $d=2$ then the equation (76) at $\tau=\infty$ (with the velocity covariance (10)) reads (here $\rho=\left|\mathbf{x}_{1}-\mathbf{x}_{2}\right|$ )

$$
\left(\mu^{2} \frac{1}{\rho} \partial_{\rho} \rho \partial_{\rho}-\mu^{2} p^{2}-p^{2} \rho^{2 \beta}\right) \mathcal{T}_{\infty}^{(2)}(\rho, p ; \mu)=\tilde{m}(\rho, p)
$$

where we defined

$$
\mathcal{S}^{(2)}\left(\rho, p_{1}, p_{2} ; \mu\right)=\delta\left(p_{1}+p_{2}\right) \mathcal{T}^{(2)}\left(\rho, p_{1} ; \mu\right)
$$

In contradistinction to the spherically symmetric case [8] eq.(85) is not explicitly soluble but its asymptotic solution (61) is easy to obtain. This asymptotic behavior is the same as the limit $\mu=0$ of the solution (85)

$$
\mathcal{T}_{\infty}^{(2)}(\rho, p ; 0)=-p^{-2} \rho^{-2 \beta} \tilde{m}(\rho, p)
$$

In general, from eq.(76) the limit $\mu=0$ can be obtained inductively

$$
\begin{aligned}
& \mathcal{S}_{\infty}^{(2 n)}\left(\mathbf{x}_{1}, \ldots, \mathbf{x}_{2 n} ; \mathbf{p}_{1}, \ldots, \mathbf{p}_{2 n} ; 0\right)=\left(\frac{1}{2} D_{0}(\mathbf{0}) \sum_{j=1}^{j=2 n} \mathbf{p}_{j}^{2}-\sum_{<j, k>} \mathbf{p}_{j} D_{0}\left(\mathbf{x}_{j}-\mathbf{x}_{k}\right) \mathbf{p}_{k}\right)^{-1} \\
& \sum_{<i, l>} \delta\left(\mathbf{p}_{i}+\mathbf{p}_{l}\right) \tilde{m}\left(\mathbf{x}_{i}-\mathbf{x}_{l}, \mathbf{p}_{i}\right) \mathcal{S}_{\infty}^{(2 n-2)}(i l ; 0)
\end{aligned}
$$

The formulas for the asymptotic behavior $(61)(\alpha=1)$ and $(66)(\alpha=1, \gamma=0)$ agree with the exact solution (87). For $n=1$ the solution (82) takes the form 
(86) whereas for $n=2$ we have

$$
\begin{aligned}
& \left.\mathcal{S}_{\infty}^{(4)}\left(\mathbf{x}_{1}, \ldots, \mathbf{x}_{4} ; \mathbf{p}_{1}, \ldots, \mathbf{p}_{4} ; 0\right)=\left(\frac{1}{2} D_{0}(\mathbf{0})\right) \sum_{j=1}^{j=4} \mathbf{p}_{j}^{2}-\sum_{<j, k>} \mathbf{p}_{j} D_{0}\left(\mathbf{x}_{j}-\mathbf{x}_{k}\right) \mathbf{p}_{k}\right)^{-1} \\
& \left(\delta\left(\mathbf{p}_{1}+\mathbf{p}_{2}\right) \delta\left(\mathbf{p}_{3}+\mathbf{p}_{4}\right)\left(\mathbf{p}_{1} D_{0}(\mathbf{0}) \mathbf{p}_{1}-\mathbf{p}_{1} D_{0}\left(\mathbf{x}_{1}-\mathbf{x}_{2}\right) \mathbf{p}_{1}\right)^{-1}\right. \\
& \left.\tilde{m}\left(\mathbf{x}_{1}-\mathbf{x}_{2}, \mathbf{p}_{1}\right) \tilde{m}\left(\mathbf{x}_{3}-\mathbf{x}_{4}, \mathbf{p}_{3}\right)+\text { permut. }\right)
\end{aligned}
$$

For the scale invariant random velocity field (10) $D_{0}(\mathbf{0})=0$ and $D_{0}\left(\mathbf{x}_{j}-\mathbf{x}_{k}\right)=$ $-\left|\mathbf{x}_{j}-\mathbf{x}_{k}\right|^{2 \beta}$. It follows from eqs.(87)-(88) that the temperature correlation functions are scale invariant under scale transformations of the coordinates $\mathbf{x}_{j}$ as well as $\mathbf{p}_{j}$. When $\mu=0$ then the correlation functions $\mathcal{S}_{\infty}^{(2 n)}$ are singular at coinciding points (the limit $\mu \rightarrow 0$ has been studied earlier by other methods in $[14][15])$. The bound (40) is valid for $\mu>0$.

\section{References}

[1] B.L. Shraiman and E.D.Siggia,Nature 405,639(2000)

[2] A.N.Kolmogorov, Dokl.Akad.Nauk SSSR,30,299(1941), J.Fluid.Mech.13,82(1962)

[3] U.Frisch, Turbulence: the legacy of A.N. Kolmogorov, Cambridge Univ.Press, 1995

[4] G. Falkovich,K. Gawedzki and M. Vergassola, Rev.Mod.Phys. 73,913(2001)

[5] A. Staicu and W.van de Water,Phys.Rev.Lett.90,094501(2003)

[6] A.J.Majda J.Stat.Phys.73,515(1993)

A.J. Majda and P.R. Kramer, Phys.Rep.314,237(1999)

[7] Q. Zhang and J.Glimm, Comm.Math.Phys.146,217(1992) 
[8] R.H. Kraichnan, Phys.Fluids,11,945(1968), Phys.Rev.Lett.72,1016(1994)

[9] K. Gawedzki and A. Kupiainen,Phys.Rev.Lett.75,3834(1995)

M.Chertkov, G. Falkovich,I.Kolokolov and V.Lebedev, Phys.Rev.E52,4924(1995)

[10] H.Kesten and G. Papanicolaou, Commun. Math.Phys.65,97(1979), A. Fannjiang and G.C.Papanicolaou, Probab.Theory Rel.Fields 105,279(1996)

[11] M. Avellaneda and A.J.Majda, Commun.Math.Phys.138,339(1991)

[12] A. Fannjiang and T.Komorowski, Annals Appl.Probab.10,1100(2000)

[13] U.Frisch, A. Mazzino, A. Noullez and M. Vergassola, Phys.Fluids,11,2178(1999),arXiv:cond-mat/9810074

[14] G.Eyink and J. Xin, Phys.Rev.Lett.77,2674(1996), arxiv: chao-dyn/9605012

[15] V. Hakulinen, Commun.Math.Phys.235,1(2003)

[16] N. Ikeda and S. Watanabe, Stochastic Differential Equations and Diffusion Equations, North Holland,1981

[17] B.Simon, Functional Integration and Quantum Physics, Academic Press, 1979

[18] A. Pumir,Phys.Rev.E57,2914(1998)

[19] J.A. Arnowitz and D.R.Nelson, Phys.Rev.A30,1948(1984)

[20] J.P.Gollub,J.Clarke,M.Gharib, B.Lane and O.N.Mesquita, Phys.Rev.Lett.67,3507(1991) 
[21] L. Biferale, E.Calzavarini,F. Toschi and R.Tripiccione, arXiv:nlin.CD/0302036,2003

[22] M. Lesieur, Turbulence in Fluids,Kluwer,1990

[23] P. Levy, Processus Stochastiques et Mouvement Brownien, deuxieme edition, Revue et Augmentee,Paris,1965

[24] Y. Le Jan and O. Raimond, arxiv:math.PR/9909147

[25] Weinan E and E.Vanden Eiden,arxiv:nlin.CD/0003028

[26] M.Kuczma, An Introduction to Functional Equations and Inequalities, PWN,Warszawa, 1986

[27] L.D. Landau and E.M.Lifshits, Fluid Dynamics, 3rd ed.,Nauka, Moscow, 1986 (in Russian)

[28] A.M. Obukhov, Izv.Akad.Nauk SSSR, Ser.Geogr.Geophys.13,58(1949)

[29] S. Corrsin, J.Appl.Phys.22,469(1951)

[30] G.K. Batchelor, J.Fluid.Mech.5,113(1959)

[31] T. Watanabe and T. Gotoh, New Journ.Phys.6,40(2004)

[32] A. Celani, M. Cencini, M. Vergassola, E. Villermaux and D. Vincenzi, J.Fluid Mech.523,99(2005), arxiv:nlin.CD/0410007

[33] L. Mydlarski, A. Pumir, B.I.Shraiman, E.D. Siggia and Z. Wahrhaft, Phys.Rev.Lett.81,4373(1998)

[34] R. Antonia, E. Hopfinger, Y. Gagne and F. Anselmet, Phys.Rev.A30,2704(1984) 
[35] D. Forster, D.R. Nelson and M.J. Stephen, Phys.Rev.A16,732(1977)

[36] K. Gawedzki and A. Kupiainen, Universality in turbulence:an exactly soluble model in Low Dimensional Models in Statistical Physics and Quantum Field Theory, H.Grosse and L.Pittner,eds., Springer,Berlin,1996, arxiv:chao-dyn/9512006

[37] A.L.Fairhall, O.Gat,V. Lvov and I.Procaccia, Phys.Rev.E53,3518(1996) 